\title{
Interactivity vs. Fairness in Networked Linux Systems
}

\author{
Wenji Wu, Matt Crawford \\ Fermilab, MS-368, Batavia, IL, 60510 \\ Email: wenji@fnal.gov, crawdad@fnal.gov
}

\begin{abstract}
In general, the Linux 2.6 scheduler can ensure fairness and provide excellent interactive performance at the same time. However, our experiments and mathematical analysis have shown that the current Linux interactivity mechanism tends to incorrectly categorize non-interactive network applications as interactive, which can lead to serious fairness or starvation issues. In the extreme, a single process can unjustifiably obtain up to 95\% of the CPU! The root cause is due to the facts that: (1) network packets arrive at the receiver independently and discretely, and the "relatively fast" non-interactive network process might frequently sleep to wait for packet arrival. Though each sleep lasts for a very short period of time, the wait-for-packet sleeps occur so frequently that they lead to interactive status for the process. (2) The current Linux interactivity mechanism provides the possibility that a non-interactive network process could receive a high CPU share, and at the same time be incorrectly categorized as "interactive." In this paper, we propose and test a possible solution to address the interactivity vs. fairness problems. Experiment results have proved the effectiveness of the proposed solution.
\end{abstract}

Keywords: Linux, Process Scheduling, Interactivity, Fairness, Networking

\section{Introduction}

Over the last several years, the Linux operating system has gained wide acceptance and is deployed in many scientific and commercial environments. Compared to previous versions, Linux 2.6 has made significant performance improvements in terms of interactivity, fairness, and scalability. Linux 2.6 is now preemptible, and has an $\mathrm{O}(1)$ CPU scheduler.

The Linux 2.6 scheduler is prioritized and epoch-based [1][2][3][4]. The whole process scheduling is based on a data structure called runqueue. A runqueue is created and maintained for each CPU in the system. The per-CPU runqueue keeps track of all runnable tasks assigned to a particular CPU. Each runqueue consists of an active priority array, and an expired priority. All runnable processes begin with the active array, and are scheduled in priority order. In general, when a process expires it is move to the expired array so that all runnable processes get an opportunity to execute. When the active array becomes empty, the expired and active arrays are switched. This unique active-expired array design is credited much to the overall system performance improvements.

One design goal of Linux 2.6 is to improve interactivity [5]. Processes such as text editors and command shells interact constantly with their users, and spend a lot of time waiting for keystrokes and mouse events. When inputs are received the process must be woken up quickly; otherwise, the user will find the system to be unresponsive and annoying. Typically, the delay must not exceed 150ms [1]. Linux 2.6 provides excellent 
interactive performance by employing the following measures [1][2][4]: (1) Its scheduler is a typical decay usage priority scheduler. Processes are scheduled in priority order, where effective priority has two constituent components: static priority and dynamic priority bonus. The static priority reflects inherent relative importance of processes, which is expressed by processes' nice values. The dynamic priority bonus depends on CPU usage patterns; the scheduler favors interactive processes and penalizes non-interactive processes by adjusting the dynamic priority bonus. (2) To reduce scheduling latency, expired interactive processes are reinserted back into the active array, instead of the expired array. In addition, an interactive processes' timeslice is divided into smaller pieces, preventing interactive processes from blocking each other. (3) Linux 2.6 is kernelpreemptible. Whenever a scheduler clock tick or interrupt occurs, if a higher-priority task has become runnable, it will preempt the running task as long as the latter holds no kernel locks. (4) Linux 2.6’s clock granularity has reached 1ms level.

Fairness is another design goal of Linux 2.6 [5]. Fairness is the ability of all tasks not only to make forward progress, but to do so relatively evenly. The opposite of fairness is starvation, which occurs if some tasks make no forward progress at all [6][7]. Linux 2.6 scheduler's active-expired-arrays design is supposed to ensure fairness [1][2]. However, as described above, an expired interactive process is reinserted back into the active array instead of the expired array. This leads to the possibility of starvation for the processes in the expired array if the active array continues to hold runnable processes. To circumvent the starvation issue, when the first expired process is older than some limit, expired processes are moved to the expired array without regard to their interactive status. Usually, an interactive process does not consume much CPU time because most of time it sleeps waiting for user input. In general, the Linux 2.6 scheduler can ensure fairness among processes, and provide excellent interactive performance at the same time. However, our experiment and analysis have shown that the current Linux interactivity mechanism tends to incorrectly categorize non-interactive network applications as interactive, which can lead to serious fairness or starvation issues. The current Linux interactivity mechanism allows the possibility that a non-interactive network process could consume a large CPU share, and at the same time is incorrectly categorized as interactive. Further, incorrectly labeled "interactive network applications" might block true interactive applications, resulting in degraded interactive performance.

Linux-based network end systems have been widely deployed in the High-Energy Physics (HEP) community at labs like CERN, DESY, Fermilab, and SLAC, and at many universities. At Fermilab, thousands of networked systems run Linux; these include computational farms, trigger processing farms, hierarchical storage servers, and desktop workstations. From a network performance perspective, Linux represents an opportunity since it is amenable to optimization and tuning due to its open source support and projects such as web100 and net100 that enable examination of internal state [8][9]. The performance of Linux-based network end systems is of great interest to HEP communicates and other scientific and commercial communities. In this paper, we analyze the interactivity vs. fairness issues in Networked Linux Systems. Our analysis is based on Linux kernel 2.6.14. Also, it is assumed that the NIC (Network Interface Card) device driver makes use of Linux's "New API," or NAPI [10][11], which reduces the interrupt 
load on the CPUs. The contributions of the paper are as follows: (1) We systematically study and analyze the Linux 2.6's scheduling and interactivity mechanism; (2) Our researches have pointed out that the current Linux interactivity mechanism is not effective in distinguishing non-interactive network processes from interactive network processes, and might result in serious fairness/starvation problems. Mathematical analysis and experiments results have verified our conclusions. (3) Further, we propose and test a possible solution to address the interactivity vs. fairness problems in networked Linux systems. Experiment results have proved the effectiveness of our proposed solution.

The remainder of the paper is organized as follows: In Section 2 the related researches on interactivity and fairness are presented. Section 3 analyzes Linux scheduling and interactivity mechanism. In Section 4, we investigate the interactivity vs. fairness problems in networked Linux systems through mathematical analysis. In section 5, we show experiment results to further study the problems, verifying our conclusions in Section 4. In Section 6, we propose and test a possible solution to address the interactivity vs. fairness problems in network Linux systems. And finally in section 7, we conclude the paper.

\section{Related Work}

The schedulers of Unix and its variants e.g., BSD4.3, FreeBSD, Solaris, and SVR4 [12][13][14][15], are typical decay usage priority schedulers: processes are scheduled in priority order; higher priority processes are scheduled to run first. The priorities of I/O bound processes (interactive processes) grow with time, so that when they are awakened, they have higher priority than CPU-bound processes (non-interactive processes), and are therefore scheduled to run immediately. In general, those schedulers provide excellent interactive response on general-purpose time-sharing systems for traditional interactive applications that have low CPU consumption. However, those schedulers are not effective in support of interactive multimedia applications (e.g., audio player, video player) that have high CPU usages. To address this problem, Y. Etsion et al. [16] proposed the human-centered scheduling of interactive and multimedia application on a loaded desktop. In their approach, the scheduler first estimates the "volume of user-interaction" associated with each process by monitoring relevant I/O device activity, then the scheduler uses those estimates to prioritize interactive processes, without respect to their CPU usage. However, this method might not be appropriate for some network applications.

To ensure fairness, proportional-share schedulers [17][18][19][20] are usually employed to control the relative rates at which different processes can use the processor. Over the years, different proportional-share schedulers have been proposed. In [17], C. A. Waldspurger et al. proposed the lottery scheduling to enable flexible control over the relative rates at which CPU-bound workloads consume processor time. In [18], P. Goyal et al. proposed a hierarchical CPU scheduler for multimedia operating systems, which provides protection between various classes of applications.

In [21], D. Petrou et al. proposed a hybrid lottery scheduler, which aims to achieve responsiveness comparable to the FreeBSD scheduler while maintaining lottery 
scheduling's flexible control over relative execution rates and load insulation. So far, no research has been found to related interactivity and fairness to network applications.

\section{Linux Scheduling and Interactivity}

Linux 2.6 is a preemptive multi-processing operating system. Processes (tasks) are scheduled to run in a prioritized round robin manner [1][2][3][4], to achieve the objectives of fairness, interactivity and efficiency. For the sake of scheduling, a Linux process has a dynamic priority and a static priority. A process' static priority is equivalent to its nice value, which is specified by user and not changed by the kernel. The dynamic priority is used by the scheduler to rate the process with respect to the other processes in the system. An eligible process with better (smaller-valued) dynamic priority is scheduled to run before a process with a worse (higher-valued) dynamic priority. The dynamic priority varies during a process' life. It depends on the process' scheduling history and its specified static priority, which we will elaborate in the following sections. There are 140 possible priority levels for processes (both dynamic priority and static priority) in Linux. The top 100 levels are used only for real-time processes, which we do not address in this paper. The last 40 levels are used for conventional processes.

\subsection{Linux Scheduler}

As shown in Figure 1, the whole process scheduling is based on a data structure called runqueue. Essentially, a runqueue keeps track of all runnable tasks assigned to a particular CPU. One runqueue is created and maintained for each CPU in a system. Each runqueue contains two priority arrays: active priority array and expired priority array. Each priority array contains a queue of runnable processes per priority level. Higher priority $(d y-$ namic priority) processes are scheduled to run first. Within a given priority, processes are scheduled round robin. All tasks on a CPU begin in the

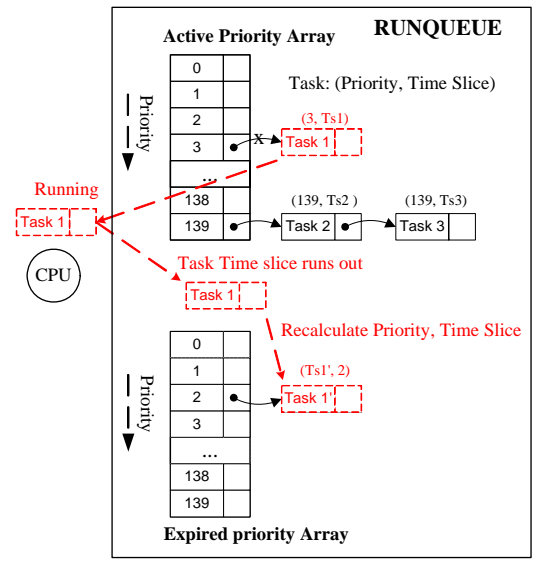

Figure 1 Linux Process Scheduling active priority array. Each process' timeslice is calculated based on its static priority; when a process in the active priority array uses up its timeslice, it is considered expired. An expired process is moved to the expired priority array if it is not interactive. An expired interactive process is reinserted into the active array if possible. In either case, a new timeslice and priority are calculated. When there are no more runnable tasks in the active priority array, it is simply swapped with the expired priority array. An unexpired process might be put into a wait queue to sleep, waiting for expected events such as completion of $\mathrm{I} / \mathrm{O}$. When a sleeping process wakes up, its timeslice and priority are recalculated and it is moved to the active priority array. As for preemption, whenever a scheduler clock tick or interrupt occurs, if a higher-priority task has become runnable, it will preempt the running task as long as the latter holds no kernel locks.

\subsection{Interactive Scheduling}


As we have said above, an interactive process needs to be responsive. The Linux kernel must provide the capabilities of interactive scheduling. To this end, it needs to:

- Perform process classification: differentiate interactive process from noninteractive process.

- Try to minimize the scheduling latency [7] for interactive process.

o Prevent non-interactive processes from blocking interactive processes.

o Prevent interactive processes from blocking other interactive processes.

The interactivity estimator is designed to find which processes are interactive and which are not. It is based on the premise that non-interactive processes tend to use up all the CPU time offered to them, whereas interactive processes often sleep [1]. A sleep_avg is stored for each process: a process is credited for its sleep time and penalized for its runtime. A process with high sleep_avg is considered interactive, and low sleep_avg is noninteractive. The interactive estimator framework embedded into Linux operates automatically and transparently.

A process' dynamic priority varies during the process' life span. It depends on the process' interactivity status and its specified static priority. Linux assigns a dynamic priority to process $P$ at time $t$ as follows:

$$
\begin{aligned}
& \text { dynamic_priority }(P, t)=\max \left\{100, \min \left\{\operatorname{static} \_\operatorname{priority}(P)+5-\operatorname{bonus}(P, t), 139\right\}\right\} \\
& \text { bonus }(P, t)=P->\text { sleep_avg }(t) * M A X_{-} \text {BONUS /MAX_SLEEP_AVG }
\end{aligned}
$$

The constant MAX_BONUS is 10 and MAX_SLEEP_AVG is $1000 \mathrm{~ms}$. P->sleep_avg(t) is the sleep_avg (in $\mathrm{ms}$ ) for process $\mathrm{P}$ at time $\mathrm{t}$, and it is limited to the range $0 \leq P$ - > sleep_avg $(t) \leq M A X \_S L E E P \_A V G$. Therefore, $\operatorname{bonus}(P, t)$ ranges from 0 to 10 . The quantity $5-\operatorname{bonus}(P, t)$ is also called the dynamic priority bonus. The more time a process spends sleeping, the higher the sleep_avg is, and the higher the priority boost as well.

From (1) and (2), it can be seen that Linux credits interactive processes and penalizes non-interactive processes by adjusting dynamic priority bonus. In this way, Linux allows interactive processes to preempt non-interactive processes when they have same, or nearly the same, static priorities.

When a process runs out its timeslice, the Linux kernel needs to determine its interactivity status. An expired interactive process is reinserted back into active array, instead of expired array. The interactivity threshold condition for process $\mathrm{P}$ is

$$
\operatorname{bonus}\left(P, t_{E}\right) \geq \text { static_priority }(P) / 4-23
$$

Where $t_{E}$ is the moment that process $\mathrm{P}$ expires. For process $\mathrm{P}$ with a default nice value of 0 , interactivity threshold is equivalent to: $P->$ sleep_avg $\left(t_{E}\right) \geq 700 \mathrm{~ms}$.

If and only if the condition in (3) holds, $\mathrm{P}$ is deemed interactive. Reinserting an interactive process into the active array helps to increase responsiveness. If it were not done, an 
interactive process in the expired array would have to wait for all the runnable processes in the active array to finish to regain the CPU. However, moving an expired interactive process back into the active array, instead of the expired array, might lead to the possibility of starvation for the processes in the expired array if the active array continues to hold runnable processes. To circumvent the starvation issue, special interactivity rules have been taken:

- Rule 1: If the time since the first process in the active array expired is greater than or equal to STARVATION_LIMIT $\times N R_{\text {running }}+1$, any expired processes are moved to the expired array without regard to their interactive status. Here, the constant STARVATION_LIMIT is $1000 \mathrm{~ms}$, and $N R_{\text {running }}$ is the number of processes in the runqueue.

- Rule 2: The interactivity is also ignored if a process in the expired array has a better static priority.

Furthermore, an interactive process P's timeslice is divided into smaller pieces. Each piece has the size of TIMESLICE_GRANULARITY $(P)$, which is actually a macro that yields the product of the number of CPUs in the system and a constant proportional to $\operatorname{bonus}(P, t)[1][4]$. An interactive process does not receive any less timeslice, instead a task of equal priority may preempt the running process every TIMESLICE_GRANULAIRTY $(P)$. The process is then requeued to the end of the list for its priority level. Processes at the same priority level run in round-robin fashion, so execution will rotate more frequently among interactive processes of the same priority, preventing them from blocking each other.

\subsection{Sleep_avg Scoring}

The basic idea of sleep_avg is to credit sleep time and penalize run time. However, the calculation of sleep_avg is not a simple counter up and down. The current interactive status of the process is used to weight both sleep time and run time to introduce some auto-regulation into the calculation [22]. The updating of sleep_avg occurs at the moments that: (a) A process wakes up from sleep or blocking state; (b) A process yields the $\mathrm{CPU}$ to other processes.

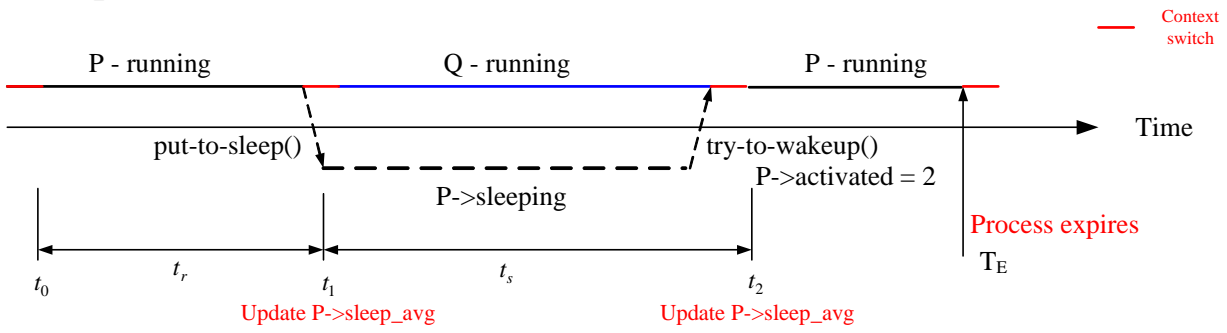

Figure 2 Updating of sleep_avg

In the example of Figure 2, at $t_{0}$ process $\mathrm{P}$ starts to run for duration of $t_{r}$. At $t_{1}, \mathrm{P}$ goes to sleep and yields the CPU to process $\mathrm{Q}$. Then at $t_{2}$, $\mathrm{P}$ wakes up and preempts $\mathrm{Q}$. In general, the updating of sleep_avg follows (4) and (5):

$P->$ sleep_avg $\left(t_{1}\right)=\max \left\{0, P->\right.$ sleep_avg $\left.\left(t_{0}\right)-t_{r} * \alpha\right\}$ 
Where $\alpha$ is a weighting factor for run time, $\alpha=1 / \max \left\{1, \operatorname{bonus}\left(P, t_{0}\right)\right\}$.

$P->$ sleep_avg $\left(t_{2}\right)=\min \left\{M A X \_S L E E P \_A V G, P->\operatorname{sleep} \_a v g\left(t_{1}\right)+t_{s}^{*} \beta\right\}$

Where $\beta$ is a weighting factor for sleep time, $\beta=\max \left\{1,10-\operatorname{bonus}\left(P, t_{1}\right)\right\}$.

However, when updating sleep_avg for waking processes, special measures are taken to treat the following scenarios [1][4]: (a) Processes that sleep a long time are categorized as idle and will get minimally interactive status to stay active, to prevent them suddenly becoming CPU intensive and starving other processes. (b) Processes waking from an uninterruptible sleep are limited in their sleep_avg rise as they are likely to have been waiting on disk $\mathrm{I} / \mathrm{O}$, which is not a strong indicator of interactivity. (Most local disk I/O is associated with uninterruptible sleep.) (c) When an awakened process is put into a runqueue, there might be scheduling latency, which could be of a non-negligible duration. In this case, the time spent on the runqueue might or might not be credited as sleep time, depending on the state of the process when it was awakened. The state of the process is encoded within the process' activated field [1]. Let's assume that Process P waits on runqueue for a period of $t_{w}$ before it is scheduled to run. The credited sleep time is as shown in Table 1. For example, a process might sleep to wait for data from network. Afterwards, when the process is woken up, its wait time on the runqueue is fully credited to the sleep_avg because its $P$ $>$ activated code is 2 .

\begin{tabular}{|c|c|c|c|c|}
\hline$P$->activated code & -1 & 1 & 2 & 0 \\
\hline Credited sleep time & 0 & $0.3^{*} t_{w}$ & $t_{w}$ & N/A \\
\hline
\end{tabular}

Table 1 Credited sleep time vs. wait time on runqueue

Since Linux only counts time in integral tick units, the Linux clock granularity might play a role when updating the sleep_avg: some sleep/run times are rounded up to the next whole tick, while others are rounded down. On average, these two effects tend to cancel out [23]. Furthermore, in Linux 2.6 the clock granularity is $1 \mathrm{~ms}$ level. In general, the sleep_avg is updated with reasonable accuracy.

\section{Interactivity vs. Fairness in Networked Linux System}

In previous sections we have discussed the Linux interactive scheduling mechanism: an expired interactive process is reinserted back into the active array, instead of the expired array. Interactive scheduling makes the Linux systems more responsive and interactive. However, interactive scheduling would bring the possibility of unfairness if the interactivity classification were inaccurate. For example, when a non-interactive process is incorrectly classified as interactive, reinserting it back into active array will gain it extra scheduling runs, at the expense of other non-interactive processes. What's worse is that when a non-interactive process might incorrectly gain "interactive status", its dynamic priority is correspondingly enhanced, which might block some true interactive processes.

As remarked above, special measures have been taken to make interactivity classification accurate. Those measures are effective in preventing processes that mainly wait for disk I/O from being categorized as interactive [22]. However, our experiments and analysis have shown that the current interactivity classification mechanism is not effective in classifying network-related processes. It tends to classify network application like ftp and 
sifying network-related processes. It tends to classify network application like ftp and rcp as interactive when bandwidth is limited or the sender is slower than the receiver. Applications like ssh, telnet, and http clients are generally interactive applications; but ftp, rcp, scp, and the like are not. If they are misclassified, it will raise scheduling fairness issues. In the following sections, we use a simplified model to analyze the fairness vs. interactivity issues in networked Linux systems.

Assume there is bulk data flowing from a sender to a receiver (as in ftp, for example). Process $\mathrm{P}$ is the data receiving process in the receiver. The network is relatively stable, and incoming packets are evenly spaced with a rate of $N_{i}$ packets/second (pps). There is no other traffic directed to the receiver. This assumption holds for traffic patterns like voice over IP [24] or an ideal TCP self-clocking stream such as in [25]. In reality, the incoming traffic pattern is irregular. However, NAPI or "Interrupt Coalescing" will mask the arrival pattern and to some extent nullify its effect on the receiver. Similar conclusions are still expected to be valid, and are borne out by experiment. Also, let the NAPI driver's hardware interrupt time be $T_{\text {int }}$, which includes NIC interrupt dispatch and service time; the software interrupt softnet's packet service rate be $R_{s n}$ (pps); and process P's data service rate is $S_{P}$ (pps). When the network bandwidth is limited, or the sender's processing power is relatively slower than the receiver's processing power, we can assume that $N_{i}<<R_{s n}$. Let process $\mathrm{P}$ have the default nice value of 0 .

\subsection{Single process receiver}

Only process $\mathrm{P}$ runs on the receiver, no other processes. At time $0, \mathrm{P}$ is waiting for network data from the sender (TCP or UDP).

As shown in Figure 3, packets start to arrive at receiver at time 0. As an interrupt-driven operating system, the Linux execution sequence is: Hardware interrupts $\rightarrow$ Software interrupts $\rightarrow$ Processes [1][2]. Packet 1 is first transferred to ring buffer, then the NIC raises a hardware interrupt to schedule softirq - softnet. Afterwards, the software interrupt handler (softnet) starts to move packet 1 from ring buffer to the socket's receive buffer of process $\mathrm{P}$, waking up process $\mathrm{P}$ and putting it to the runqueue. During this period, new packets might arrive at the receiver. For example, packet 2 arrives during the period in Figure 3. Softnet continue to process the packets within the ring buffer till it is empty. Letting $T_{s n}$ be the duration that Softnet spends on the ring buffer, we see that

$$
1+\left\lfloor\left(T_{\text {int } r}+T_{s n}\right) * N_{i}\right\rfloor=T_{s n} * R_{s n}
$$

Here, $T_{s n} * R_{s n}$ is actually the number of packets that are handled together.

$$
T_{s n}=\left\lfloor\frac{1+T_{\text {intr }} * N_{i}}{R_{s n}-N_{i}} * R_{s n}\right\rfloor / R_{s n}
$$

Then softirq yields the CPU. Process P begins to run, moving data from the socket's receive buffer into user space. Since there are $T_{s n} * R_{s n}$ packets in the receiver buffer, 
process $\mathrm{P}$ runs for a duration of $T_{r}=\left(T_{s n} * R_{s n}\right) / S_{P}$. Here, we are considering a relatively low packet-incoming rate compared to the receiver's processing power. Before the next packet (P3 in Figure 3) arrives at the receiver, process P runs out of data, and again goes to sleep, waiting for more. Either of the following conditions could lead to a relatively low incoming packet rate: (1) the network bandwidth from sender to receiver is low; (2) the sender's hardware is less powerful than the receiver's. If the next packet arrives before process $\mathrm{P}$ goes to sleep, the sender will overrun the receiver. Incoming packets would accumulate in the socket's receive buffer. For TCP traffic, the flow control mechanism would take effect to slow down the sender.

When the next packet arrives at the receiver, the same scenario as described above occurs. And the cycle repeats itself till process $\mathrm{P}$ stops. At time $t_{E}$, process P's timeslice expires.

When incoming traffic wakes up process $\mathrm{P}$, its wait time on runqueue is fully credited to the sleep_avg. For the process being discussed, its P->activation code is 2 . As shown in Figure 3 process $\mathrm{P}$ runs for $T_{r}$, and sleeps for $T_{s}$ in each cycle.

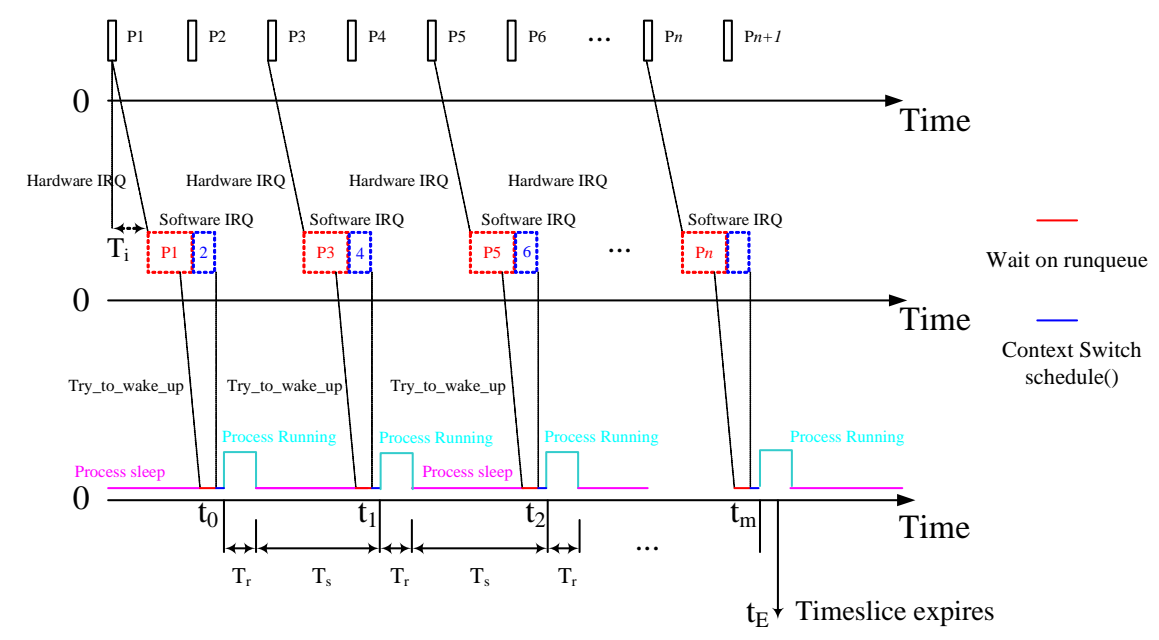

Figure 3 Interactivity vs. Fairness in Networked Linux

Here:

$$
\begin{aligned}
& T_{r}=\frac{T_{s n} * R_{s n}}{S_{P}}=\frac{\left\lfloor\frac{1+T_{\text {intr }} * N_{i}}{R_{s n}-N_{i}} * R_{s n}\right\rfloor}{S_{P}} \\
& T_{s}=\frac{\left\lfloor\frac{1+T_{\text {intr }} * N_{i}}{R_{s n}-N_{i}} * R_{s n}\right\rfloor}{N_{i}}-\frac{\left\lfloor\frac{1+T_{\text {intr }} * N_{i}}{R_{s n}-N_{i}} * R_{s n}\right\rfloor}{S_{P}}
\end{aligned}
$$

Following (4) and (5), it is easy to update $P->$ sleep_avg(t) at time $t$. 
From (8) and (9), it can be easily derived that:

$$
\frac{T_{r}}{T_{s}}=\frac{N_{i}}{S_{P}-N_{i}}
$$

Correspondingly, process P's CPU share is:

$$
\frac{T_{r}}{T_{r}+T_{s}}=\frac{N_{i}}{S_{P}}
$$

Given the receiver and process $\mathrm{P}, S_{P}$ is correspondingly fixed. Therefore, it can be derived from (3), (4), (5), and (10) that process P's interactivity status would be strongly dependent on the packet arrival rate $N_{i}$, instead of interactive activities.

As shown in Figure 3, we will count cycles of run and sleep beginning when the process wakes up. Cycle 1 starts at $t_{0}$ and ends at $t_{1}$. Since an interval $T_{r}$ of running is not more than 100ms and decreases sleep_avg by $\alpha T_{r}$, with $\alpha \leq 1$, sleep_avg may fall to the next $100 \mathrm{~ms}$ bracket during the running portion of a cycle, but no further. This may increase $\beta$ by 1 , but no more. Referring to (4) and (5), we collect the possible changes of sleep_avg in one cycle, $\Delta$ sleep_avg, in Table 2.

\begin{tabular}{|c|c|c|c|}
\hline$P->$ sleep_avg $\left(t_{0}\right)$ & $\alpha$ & $\beta$ & $\Delta$ sleep_avg \\
\hline $0 \leq P->$ sleep_avg $\left(t_{0}\right)<100$ & 1 & 10 & $10 T_{s}-T_{r}$ \\
\hline $100 \leq P->$ sleep_avg $\left(t_{0}\right)<200$ & 1 & 10 or 9 & $10 T_{s}-T_{r}$ or $9 T_{s}-T_{r}$ \\
\hline $200 \leq P->$ sleep_avg $\left(t_{0}\right)<300$ & $1 / 2$ & 9 or 8 & $9 T_{s}-T_{r} / 2$ or $8 T_{s}-T_{r} / 2$ \\
\hline $300 \leq P->$ sleep_avg $\left(t_{0}\right)<400$ & $1 / 3$ & 8 or 7 & $8 T_{s}-T_{r} / 3$ or $7 T_{s}-T_{r} / 3$ \\
\hline $400 \leq P->$ sleep_avg $\left(t_{0}\right)<500$ & $1 / 4$ & 7 or 6 & $7 T_{s}-T_{r} / 4$ or $6 T_{s}-T_{r} / 4$ \\
\hline $500 \leq P->$ sleep_avg $\left(t_{0}\right)<600$ & $1 / 5$ & 6 or 5 & $6 T_{s}-T_{r} / 5$ or $5 T_{s}-T_{r} / 5$ \\
\hline $600 \leq P->$ sleep_avg $\left(t_{0}\right)<700$ & $1 / 6$ & 5 or 4 & $5 T_{s}-T_{r} / 6$ or $4 T_{s}-T_{r} / 6$ \\
\hline $700 \leq P->$ sleep_avg $\left(t_{0}\right)<800$ & $1 / 7$ & 4 or 3 & $4 T_{s}-T_{r} / 7$ or $3 T_{s}-T_{r} / 7$ \\
\hline $800 \leq P->$ sleep_avg $\left(t_{0}\right)<900$ & $1 / 8$ & 3 or 2 & $3 T_{s}-T_{r} / 8$ or $2 T_{s}-T_{r} / 8$ \\
\hline $900 \leq P->$ sleep_avg $\left(t_{0}\right)<1000$ & $1 / 9$ & 2 or 1 & $2 T_{s}-T_{r} / 9$ or $T_{s}-T_{r} / 9$ \\
\hline$P->$ sleep_avg $\left(t_{0}\right)=1000$ & $1 / 10$ & 1 & $T_{s}-T_{r} / 10$ \\
\hline
\end{tabular}

Table 2 Changes of sleep_avg in each cycle

From Table 2, we can have the following theorem.

Theorem 1: Process $\mathrm{P}$ is the data receiving process in the receiver. The network is relatively stable, and incoming packets are evenly spaced with a rate of $N_{i}$ (pps). And Process P's data service rate is $S_{P}$ (pps). If $N_{i} / S_{P}<0.9$, P will be categorized as "interactive" if it runs long enough.

Proof: If $N_{i} / S_{P}<0.9$, from (10) it can be derived that $T_{r} / T_{s}<9$. From table 2, it can be seen that when $T_{r} / T_{s}<9$, $\Delta$ sleep_avg $>0$ for any cycle. To categorize a process as "in- 
teractive," it needs to meet the condition in (3). Let's assume process P's initial sleep_avg is sleep_avg(0) when it is initially forked, and its nice value is 0 .

- If sleep_avg(0) $\geq 700 \mathrm{~ms}$. Since $\Delta$ sleep_avg $>0$ for any cycle, process $\mathrm{P}$ will always be categorized as "interactive"

- If sleep_avg $(0)<700 m s$. Since $\Delta$ sleep_avg $\geq 4 T_{s}-T_{r} / 6>\frac{5}{2} T_{s}$ for any cycle, process $\mathrm{P}$ needs to run for some finite number of cycles $n$ to achieve $\sum_{k=1}^{n} \Delta s l e e p \_a v g(k)>700 m s-s l e e p \_a v g(0)$.

Therefore, process $\mathrm{P}$ will meet the condition in (3) to be categorized if it is running long enough.

Proof ends.

Theorem 1 shows that process P's interactivity status is strongly dependent on its packet arrival rate $N_{i}$, instead of its interactive activities. Clearly, we can make the conclusions: network packets arrive at the receiver independently and discretely and the "relatively fast” non-interactive network process might frequently sleep to wait for packet arrival. Though each sleep lasts a very short period of time, the wait-for-packet sleeps occur so frequently that they lead to interactive status for the process.

The current Linux interactivity mechanism carries the chance that a non-interactive network process could consume a high CPU share, and at the same time be incorrectly categorized as “interactive." For example, assuming 700ms $\leq P->$ sleep_avg $\left(t_{0}\right)<800 \mathrm{~ms}$, process $\mathrm{P}$ has gained interactive status. Based on Table 2, the change of sleep_avg in each cycle is $4 T_{s}-T_{r} / 7$ (or $3 T_{s}-T_{r} / 7$ ). To keep the interactive status, it needs to meet the condition of $4 T_{s}-T_{r} / 7 \geq 0$ (or $3 T_{s}-T_{r} / 7 \geq 0$ ), Which is $T_{r} / T_{s} \leq 28$ (or $T_{r} / T_{s} \leq 21$ ). This condition can be easily met in normal network conditions. However, although process $\mathrm{P}$ keeps its interactive status, process $\mathrm{P}$ might still be using a high CPU percentage. When process $\mathrm{P}$ just meets the condition of $T_{r} / T_{s} \leq 28$ (or $T_{r} / T_{s} \leq 21$ ) to keep the interactive status, its CPU can reach as high as $96.55 \%$. Table 3 shows process P's maximal CPU share at different scenarios while keeping its sleep_avg in the indicated range.

\begin{tabular}{|c|c|c|}
\hline$P->$ sleep_avg $\left(t_{0}\right)$ & $T_{r} / T_{s}$ & CPU share \\
\hline $700 \leq P->$ sleep_avg $\left(t_{0}\right)<800$ & 21 or 28 & $95.45 \%$ or $96.55 \%$ \\
\hline $800 \leq P->$ sleep_avg $\left(t_{0}\right)<900$ & 16 or 24 & $94.12 \%$ or $96 \%$ \\
\hline $900 \leq P->$ sleep_avg $\left(t_{0}\right)<1000$ & 9 or 18 & $90 \%$ or $94.74 \%$ \\
\hline$P->$ sleep_avg $\left(t_{0}\right)=1000$ & 10 & $90.91 \%$ \\
\hline
\end{tabular}

Table 3 Process P’s CPU share

\subsection{Receiver plus other CPU load}

In this case, process $\mathrm{P}$ runs on the receiver with $\mathrm{M}$ other non-interactive processes. All the processes have the same default nice value of 0 .

Theorem 2: Process $\mathrm{P}$ runs on the receiver with $\mathrm{M}$ non-interactive processes. All the processes have the default nice value of 0 . Assume that the network is relatively stable, and $\mathrm{P}$ has already gained interactive status. For process $\mathrm{P}$, if $N_{i} / S_{P}<0.9$, no matter how 
many non-interactive processes run on the system, process $\mathrm{P}$ will have a CPU share of $N_{i} / S_{P}$, rather than $1 /(M+1)$. The $\mathrm{M}$ non-interactive processes' total CPU share is: $\left(S_{P}-N_{i}\right) / S_{P}$, rather than $M /(M+1)$.

Proof: All processes begin in the active array, and are scheduled as described in section 3. Since all processes have the same nice value, hence the same static priority, the second special interactivity rule to circumvent starvation for the processes in the expired array is not applicable here. Before process $\mathrm{P}$ is moved to the expired array, process $\mathrm{P}$ will maintain its interactive status and have higher dynamic priority than the $\mathrm{M}$ non-interactive processes. This is due to the facts: (1) process $\mathrm{P}$ has already gained interactive status; (2) if it has $N_{i} / S_{P}<0.9$, it has $\Delta$ sleep_avg $>0$ for any cycle, as proven in Theorem 1 . Therefore, process $\mathrm{P}$ will not lose its interactive status. When process $\mathrm{P}$ expires, it will be reinserted in the active array, until the condition in the first special interactivity rule is satisfied.

Also, considering that Linux is preemptive: whenever a scheduler clock tick or interrupt occurs, if a higher-priority task has become runnable, it will preempt the running task as long as the latter holds no kernel locks. Therefore, no matter how many non-interactive processes run on the system, before process $\mathrm{P}$ is moved to the expired array, process P's scheduling pattern is the same as that of the scenario discussed in section 4.1, where only process $\mathrm{P}$ runs on the receiver. Correspondingly, process P's CPU share won't change: it is $N_{i} / S_{P}$. The $\mathrm{M}$ non-interactive processes' total CPU share is: $\left(S_{P}-N_{i}\right) / S_{P}$. The $\mathrm{M}$ non-interactive processes can only run while process $\mathrm{P}$ sleeps.

According to the first special interactivity rule: "if the time since the first process in the active array expires is greater than or equal to STARVATION_LIMIT $\times N R_{\text {running }}+1$, any expired processes are moved to the expired array without regard to their interactive status”. In all, there are $\mathrm{M}+1$ processes in the runqueue, which implies $N R_{\text {running }}=M+1$. Also, STARVATION_LIMIT $=1000 \mathrm{~ms}$. Let us denote the timeslice of a process with nice value $\eta$ as timeslice $(\eta)$; $\operatorname{timeslice}(0)=100 \mathrm{~ms}$.

If it the case that $N_{i} / S_{P}<0.9$, we will have: $\left(S_{P}-N_{i}\right) / S_{P}>0.1$, then it follows that STARVATION_LIMIT $\times(M+1) \times\left(S_{P}-N_{i}\right) / S_{P}>M \times$ timeslice $(0)+1$. This implies that all the $\mathrm{M}$ non-interactive processes will expire and be moved to the expired array before the first special interactivity rule comes into effect. Since non-interactive processes are running only when process $\mathrm{P}$ is sleeping, at the moment when the last non-interactive process expires and is moved to the expired array, there is no runnable process in the active array. Then the active array is switched with the expired array, and a new cycle starts. Therefore, process P's scheduling pattern is the same as that of the scenario discussed in Theorem 2. Correspondingly, process P's CPU share won't change; it is $N_{i} / S_{P}$, instead of $1 /(M+1)$. The $\mathrm{M}$ non-interactive processes can only run when process $\mathrm{P}$ is sleeping. The $\mathrm{M}$ non-interactive processes' total CPU share is $\left(S_{P}-N_{i}\right) / S_{P}$, instead of $M /(M+1)$.

Proof ends. 
From Theorem 2, it can be seen that networked Linux systems can have serious fairness problems. For example, if $\mathrm{M}$ is 10 and $N_{i} / S_{P}=0.85$, then, process P's CPU share would be as high as $85 \%$ while the total CPU shares of the 10 non-interactive process is only $15 \%$. This establishes our conclusion that the Linux interactivity mechanism carries the chance that a non-interactive network process could consume a high CPU share, and at the same time be incorrectly categorized as "interactive".

\section{Experiments and Analysis}

To verify our claims in section 4, we run data transmission experiments upon Fermilab's sub-networks, and the wide area networks between Brookhaven National Laboratory (BNL) and Fermilab (FNAL). In the experiments, we run iperf [26] to send data in one direction between two computer systems. iperf ${ }^{ \pm}$in the receiver is the data receiving process P. The sub-networks used at Fermilab are as shown in Figure 4a. The sender and receiver are attached to two Cisco 6509 switches connected to each other by an uncongested 10-gigabit/second link. During the experiments, the background traffic in the network is low, and there is no packet loss or reordering in the network. For the network, the Round Trip Time (RTT) statistics are: $\mathrm{min} / \mathrm{avg} / \mathrm{max} / \mathrm{dev}=0.134 / 0.146 / 0.221 / 0.25 \mathrm{~ms}$. In the experiments on local subnets, we use two different senders, one more powerful than the other. For simplicity, in the following sections, they are termed "Fast Sender" and "Slow Sender" respectively. The sender and receiver's characteristics are shown in table 4. Here, the "Fast Sender" and "Slow Sender" are relative to each other. At their full transmission capacities, both senders can saturate the Gigabit Ethernets.

The wide area networks between BNL and FNAL are as shown in Figure 4b. During the experiments, data are transmitted from BNL to FNAL. There might be packet loss, or packet reordering in the wide area networks. The senders and receiver's characteristics are shown in table 5 . The receiver is the same system as the one used on local subnets. For the network, the RTT statistics are: $\mathrm{min} / \mathrm{avg} / \mathrm{max} / \mathrm{dev}=23.563 / 23.633 / 23.773 / 0.172$ ms.

\begin{tabular}{|c|c|c|c|}
\hline & Fast Sender & Slow Sender & Receiver \\
\hline CPU & $\begin{array}{c}\text { Two Intel Xeon CPUs } \\
(3.0 \mathrm{GHz})\end{array}$ & $\begin{array}{c}\text { One Intel Pentium IV } \\
\text { CPU }(2.8 \mathrm{GHz})\end{array}$ & $\begin{array}{c}\text { One Intel Pentium III } \\
\text { CPU (1 GHz) }\end{array}$ \\
\hline System Memory & $3829 \mathrm{MB}$ & $512 \mathrm{MB}$ & $512 \mathrm{MB}$ \\
\hline \multirow{2}{*}{ NIC } & $\begin{array}{c}\text { Syskonnect, 32bit-PCI } \\
\text { bus slot at 33MHz, } \\
1 \text { Gbps, twisted pair }\end{array}$ & $\begin{array}{c}\text { Intel PRO/1000, 32bit- } \\
\text { PCI bus slot at 33 MHz } \\
1 \mathrm{Gbps} \text { twisted pair }\end{array}$ & $\begin{array}{c}\text { 3COM, 3C996B-T, 32bit- } \\
\text { PCI bus slot at 33MHz, } \\
1 \mathrm{Gbps} \text {, twisted pair }\end{array}$ \\
\hline
\end{tabular}

Table 4 Senders and Receiver Features for Experiments upon Fermilab’s Sub-networks

\begin{tabular}{|c|c|c|}
\hline & BNL Sender & FNAL Receiver \\
\hline CPU & One Intel Pentium IV CPU (3.2 GHz) & One Intel Pentium III CPU (1 GHz) \\
\hline System Memory & $1 \mathrm{G}$ & $512 \mathrm{MB}$ \\
\hline \multirow{2}{*}{ NIC } & Intel PRO/1000, 32bit-PCI bus slot at & 3COM, 3C996B-T, 32bit-PCI bus slot at \\
& 33MHz, 1Gbps, twisted pair & 33MHz, 1Gbps, twisted pair \\
\hline
\end{tabular}

Table 5 Sender and Receiver Features for Experiments upon Wide Area Networks

${ }^{ \pm}$Iperf is multi-threaded; here we mean the iperf data transmission/reception thread. 


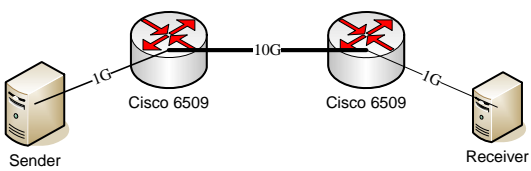

Figure 4a Fermilab Sub-Networks

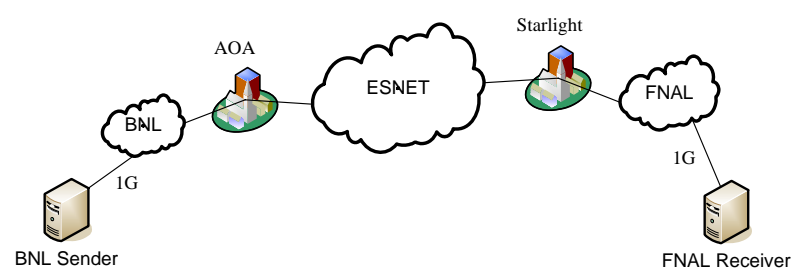

Figure 4b Wide Area Networks between BNL and FNAL

In order to study the detailed interactive scheduling process, we have added instrumentation within Linux kernel. Specifically, (1) we keep track of the sleep_avg for each process at the moments its timeslice runs out; (2) we monitor the number of times that a process is reinserted into the active array due to its interactive status. For simplicity, it is termed "reinsertion count;" (3) we collect each process' stime and utime $\boldsymbol{t}^{+}$when it is terminated. Also to study the effects of interactive scheduling on system performance, we create a non-interactive scheduling Linux, in which expired processes are inserted into the expired array, without regard to their interactivity status. In the following sections, we term "WI" for interactive scheduling, and "NI" for non-interactivity scheduling.

To create non-interactive processes in the receiver, we run a purely CPU intensive application that executes a number of arithmetic operations in a loop. Non-interactive processes run as background loads. If there are $m$ such processes in the receiver, it is termed as "BLm". In all the experiments, the sender transmits one TCP stream to the receiver for 100 seconds. In the receiver, iperf is run as "iperf $\boldsymbol{- s}-\boldsymbol{w} \mathbf{2 0 M}$ ". All the processes are running with a nice value of 0 . Further, since the transmission lasts 100 seconds, in the receiver we calculate iperf's CPU share as: (stime + utime)/100s. Consistent results were obtained across repeated runs. In the following sections, we present our experiment results.

\subsection{Experiments over local subnets}

Table 6 and 7 shows the iperf experiment results in the receiver for both slow sender and fast sender. In the experiments, the background loads are varied. For each group of data in the tables, we run the same experiments five times, and choose the group of data with highest transmission throughput. The corresponding experiment results for iperf in the receiver are recorded. Those data include throughput, iperf CPU shares, and reinsertion count. Also, in the experiments, we compare interactive to non-interactive scheduling. Iperf itself is not an interactive application. However, the experiment results in Table 6 and 7 show that iperf's interactive status is strongly dependent on the network conditions: iperf is more readily categorized as interactive with a slow sender than a fast sender. This verifies our claims in Section 4: when network packets arrive at the receiver independently and discretely, the "relatively fast" non-interactive network process might frequently sleep to wait for packet arrival. Though each sleep lasts a very short period of time, the wait-for-packet sleeps occur so frequently that they lead to interactive status for the process.

\footnotetext{
${ }^{+}$stime, utime: the time process spent in the kernel space and user space respectively.
} 


\begin{tabular}{|c|c|c|c|c|}
\hline Load & Scheduler & Throughput & $\begin{array}{c}\text { CPU } \\
\text { Share }\end{array}$ & $\begin{array}{c}\text { Reinsertion } \\
\text { Count }\end{array}$ \\
\hline \multirow{2}{*}{ BL0 } & WI & $436 \mathrm{Mbps}$ & $78.489 \%$ & 780 \\
\cline { 2 - 5 } & NI & $473 \mathrm{Mbps}$ & $87.569 \%$ & 0 \\
\hline \multirow{2}{*}{ BL1 } & WI & $443 \mathrm{Mbps}$ & $81.573 \%$ & 815 \\
\cline { 2 - 5 } & NI & $285 \mathrm{Mbps}$ & $49.923 \%$ & 0 \\
\hline \multirow{2}{*}{ BL2 } & WI & $438 \mathrm{Mbps}$ & $80.613 \%$ & 801 \\
\cline { 2 - 5 } & NI & $185 \mathrm{Mbps}$ & $33.022 \%$ & 0 \\
\hline \multirow{2}{*}{ BL4 } & WI & $430 \mathrm{Mbps}$ & $79.217 \%$ & 785 \\
\cline { 2 - 5 } & NI & $113 \mathrm{Mbps}$ & $20.025 \%$ & 0 \\
\hline \multirow{2}{*}{ BL8 } & WI & $440 \mathrm{Mbps}$ & $81.093 \%$ & 811 \\
\cline { 2 - 5 } & NI & $64.7 \mathrm{Mbps}$ & $11.117 \%$ & 0 \\
\hline
\end{tabular}

Table 6 Iperf Experiment Results in the Receiver (Slow Sender)

\begin{tabular}{|c|c|c|c|c|}
\hline Load & Scheduler & Throughput & $\begin{array}{c}\text { CPU } \\
\text { Share }\end{array}$ & $\begin{array}{c}\text { Reinsertion } \\
\text { Count }\end{array}$ \\
\hline \multirow{2}{*}{ BL0 } & WI & $464 \mathrm{Mbps}$ & $99.228 \%$ & 7 \\
\cline { 2 - 5 } & NI & $478 \mathrm{Mbps}$ & $99.975 \%$ & 0 \\
\hline \multirow{2}{*}{ BL1 } & WI & $241 \mathrm{Mbps}$ & $49.995 \%$ & 7 \\
\cline { 2 - 5 } & NI & $241 \mathrm{Mbps}$ & $50.197 \%$ & 0 \\
\hline \multirow{2}{*}{ BL2 } & WI & $159 \mathrm{Mbps}$ & $34.246 \%$ & 8 \\
\cline { 2 - 5 } & NI & $160 \mathrm{Mbps}$ & $32.826 \%$ & 0 \\
\hline \multirow{2}{*}{ BL4 } & WI & $97.0 \mathrm{Mbps}$ & $20.859 \%$ & 8 \\
\cline { 2 - 5 } & NI & $105 \mathrm{Mbps}$ & $20.175 \%$ & 0 \\
\hline \multirow{2}{*}{ BL8 } & WI & $74.2 \mathrm{Mbps}$ & $15.375 \%$ & 47 \\
\cline { 2 - 5 } & NI & $58.3 \mathrm{Mbps}$ & $11.143 \%$ & 0 \\
\hline
\end{tabular}

Table 7 Iperf Experiment Results in the Receiver (Fast Sender)

For better comparison and presentation, we show the reinsertion count of different experiment scenarios in Figure 5. In the case of slow sender, the reinsertion count is around 800 at different background loads; as for fast sender, the highest reinsertion count is only 47. As the experiment runs for 100 seconds, and the timeslice for a process with default nice value of 0 is $100 \mathrm{~ms}$, there cannot be more than 1000 expirations of iperf's timeslice. When eliminating factors of process sleep time and system interrupt time by noting iperf's CPU share, reinsertion count of 800 implies that iperf is categorized as interactive almost all the time.

Experiment results in Table 6 and 7 also verify the correctness of Theorem 2: interactive scheduling can lead to the fairness issue. As for non-interactive scheduling, when the number of background processes increases, iperf's CPU share is correspondingly reduced. Basically, if the $\mathrm{M}+1$ processes run in the system, each process has its share of $1 /(M+1)$. However, under interactive scheduling, iperf's CPU shares are dependent on the network conditions. With a slow sender, iperf's CPU shares keeps around 80\%, no matter how many background processes there are. This is in accord with Theorem 2 . With a fast sender, iperf's CPU share is similar to what it receives under non-interactive scheduling. For better presentation, we show the results of CPU shares in Figure 6. In the Figure, “FWI" represents fast sender and interactive scheduling in the receiver; "SWI" represents slow sender and interactive scheduling in the receiver; "FNI" represents fast sender 
and non-interactive scheduling in the receiver; "SNI" represents slow sender and noninteractive scheduling in the receiver.

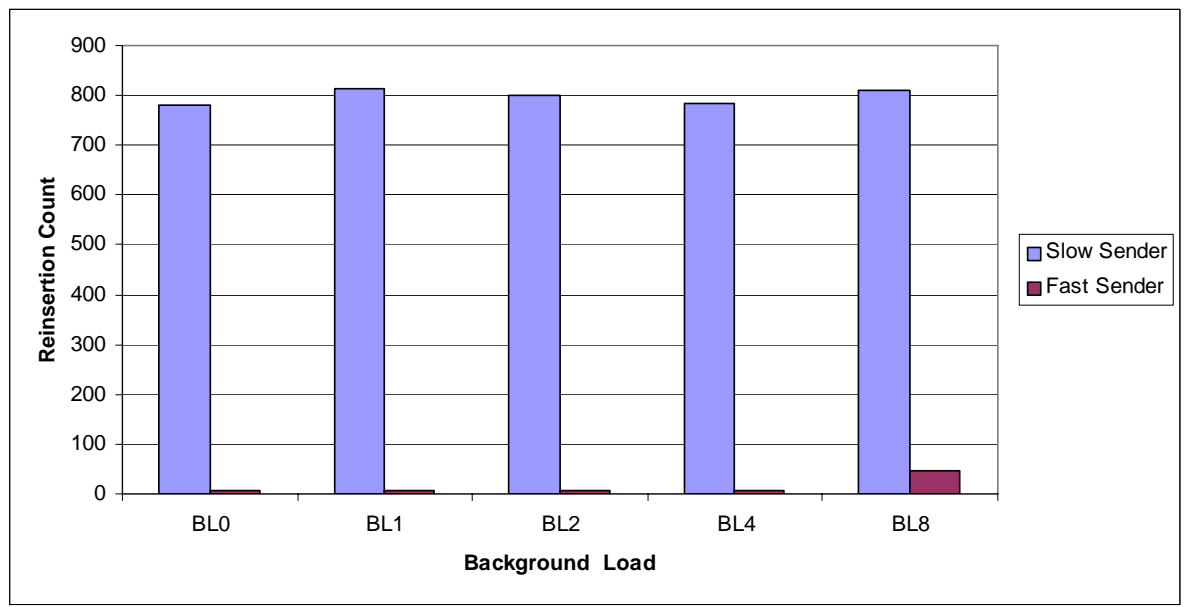

Figure 5 Comparison of "Reinsertion Count”

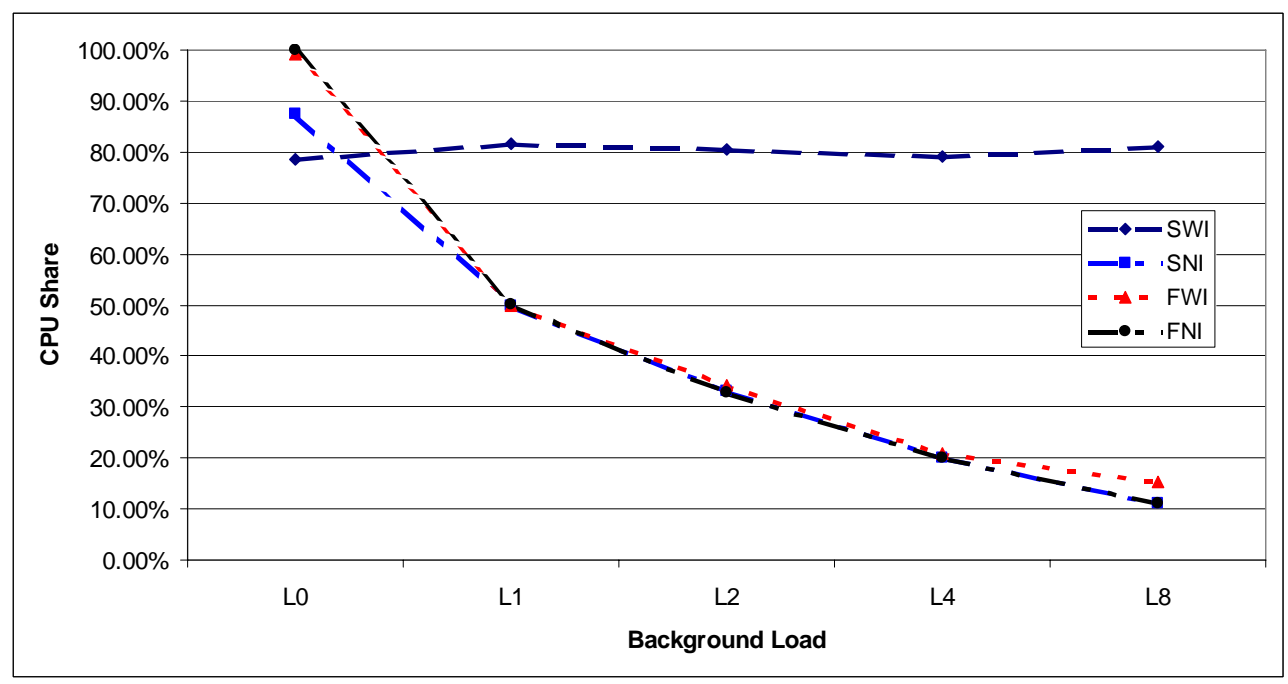

Figure 6 Comparisons of CPU Shares

To further probe the interactivity vs. fairness issue, we randomly choose two groups of experiment results. The experiments are run with background load of BL8, one with fast sender, and the other with slow sender. The experiments results are given in Figures 7 10 .

Figure 7 and 8 give iperf's sleep_avg in the receiver for slow and fast sender respectively. For the slow sender (Figure 7), it can be seen that iperf's sleep_avg is always greater than 700ms. It means that iperf is categorized as "interactive" all the time. However, for the fast sender (Figure 8), iperf is categorized as non-interactive most of the time. This is the reason that with a fast sender, iperf's CPU shares are similar with those of non-interactive scheduling. These experiment results agree with our analysis in previous sections. It further demonstrates that the current interactivity classification mechanism is not effective 
in classifying network-related processes, which are strongly dependent on the network conditions.

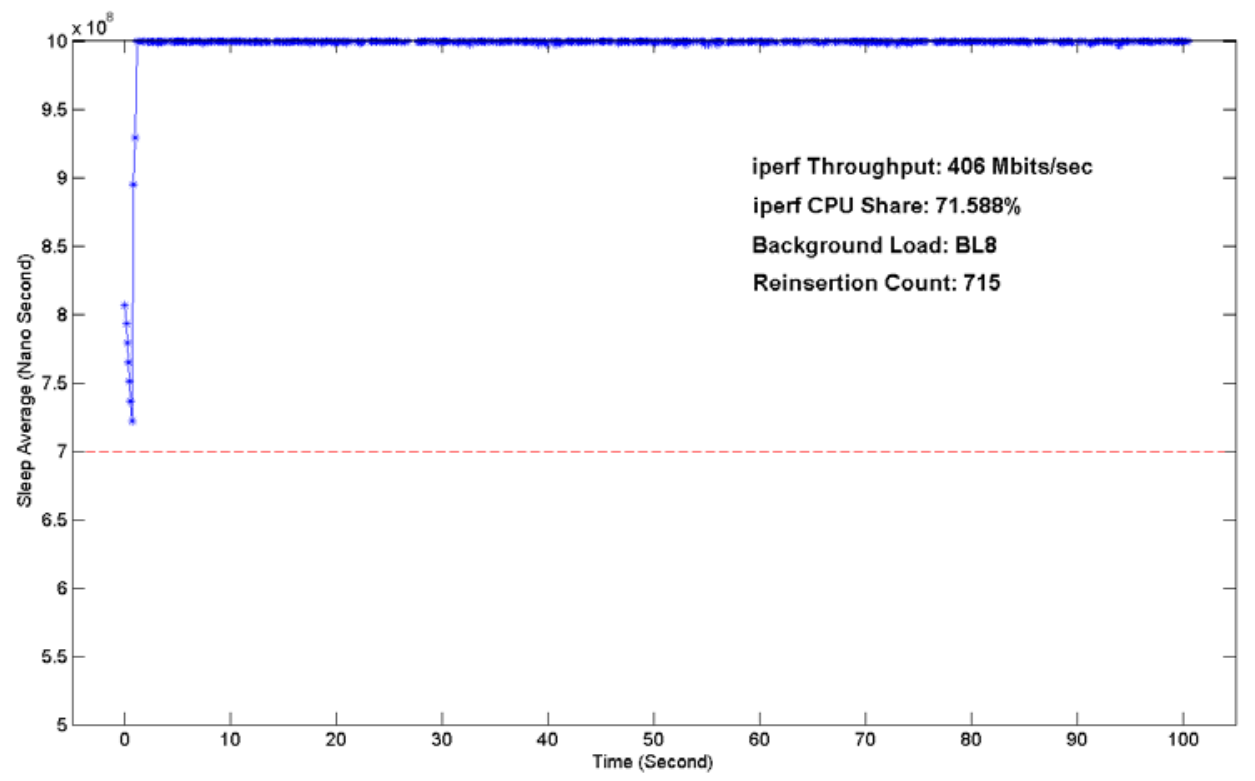

Figure 7 Iperf's sleep_avg in the Receiver (Slow Sender)

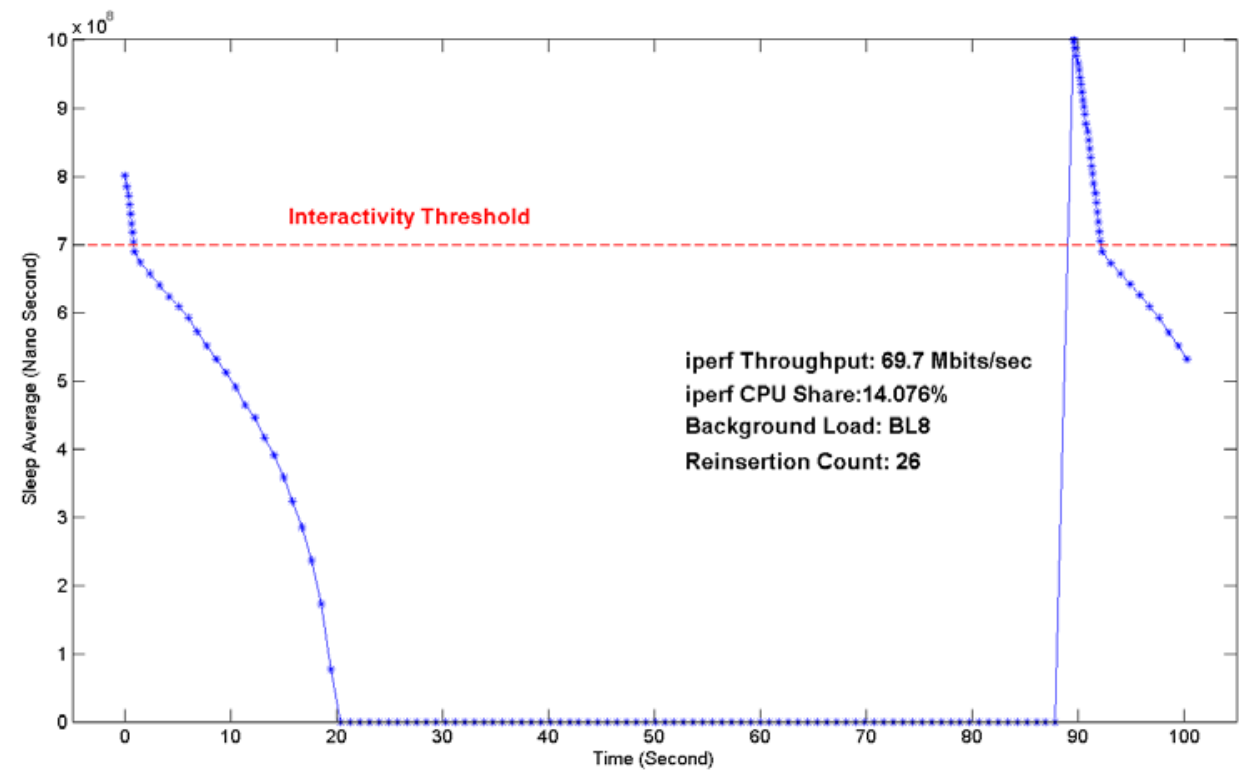

Figure 8 Iperf's sleep_avg in the Receiver (Fast Sender) 


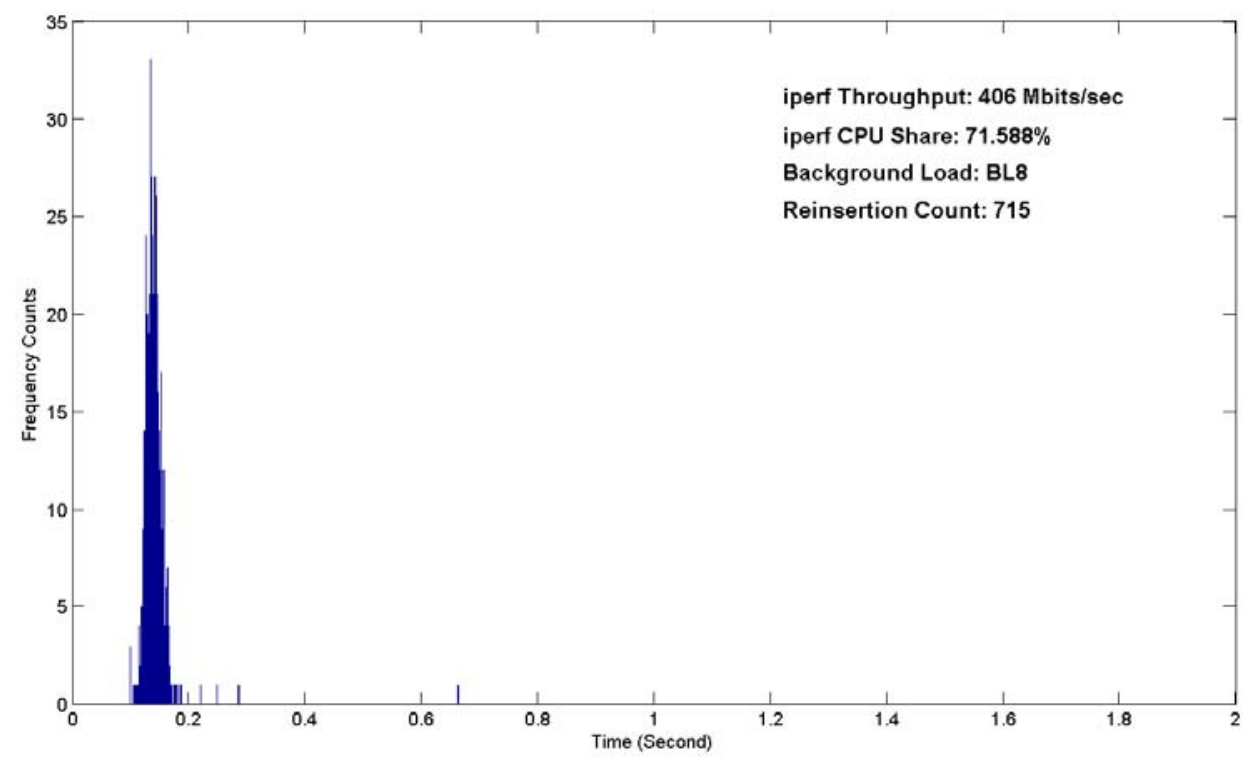

Figure 9 Histogram of Time Intervals between Consecutive Timeslice Expiration Instants for Iperf in the Receiver (Slow Sender)

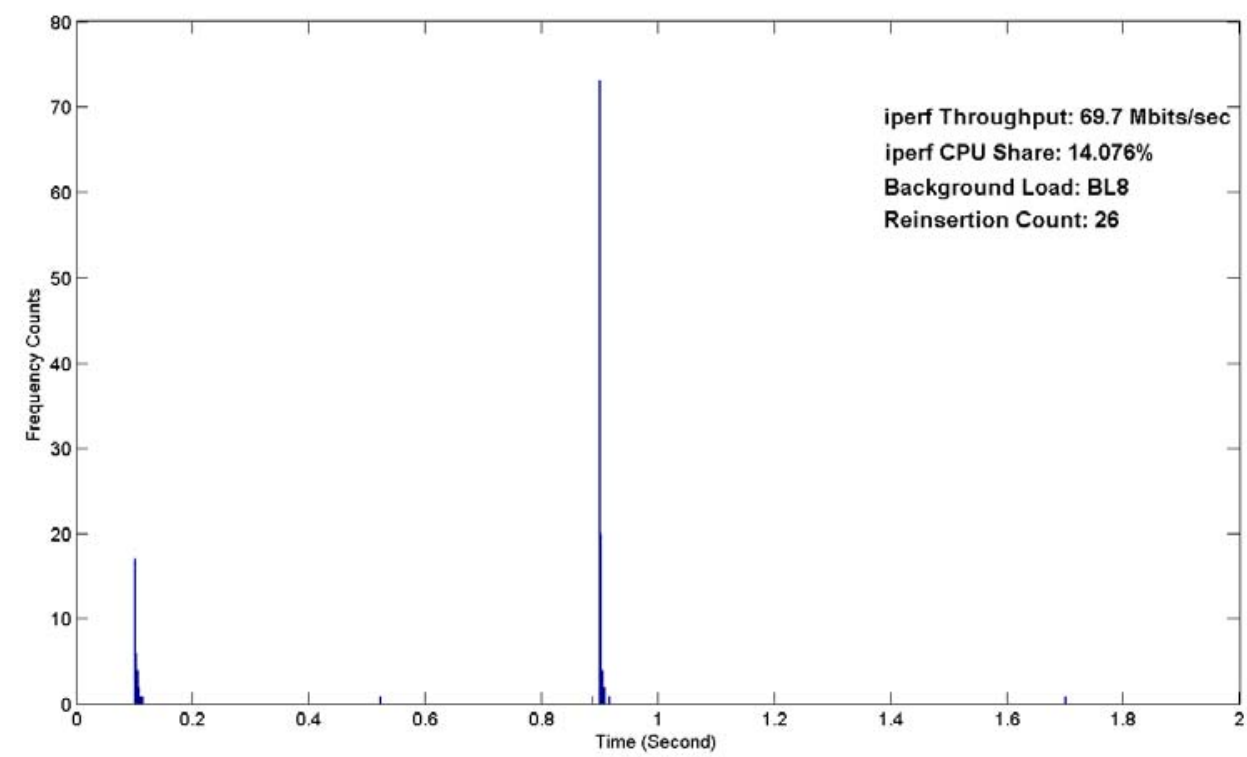

Figure 10 Histogram of Time Intervals between Consecutive Timeslice Expiration Instants for Iperf in the Receiver (Fast Sender)

Figures 9 and 10 give the histograms of time intervals between consecutive timeslice expiration instants for iperf in the receiver. These results verify the correctness of Theorem 2 from another perspective. It has been shown in Figure 7 that with the slow sender iperf is always categorized as "interactive". Therefore, each time when iperf's timeslice expires, it is reinserted into the active array, instead of the expired array. Also, due to its interactive status, iperf gains a priority bonus, resulting in higher dynamic priority than other non-interactive processes. Those non-interactive processes only run during the periods that iperf sleeps. Considering that facts that (1) with a nice value of 0 , the timeslice is $100 \mathrm{~ms}$; (2) iperf might sleep to wait for data, most of the time intervals between consecutive timeslice expiration instants in Figure 9 are between $100 \mathrm{~ms}$ and $200 \mathrm{~ms}$. 
However, Figure 10, the fast sender case, shows another story. This is due to the fact that iperf is non-interactive most of time with a fast sender (Figure 8). Once iperf expires, it will be move to the expired array and can only regain the CPU after all 8 non-interactive processes finish their timeslice. That is why the majority of the time intervals between consecutive timeslice expiration instants for iperf are greater than $900 \mathrm{~ms}$.

\subsection{Experiments over Wide Area Networks from BNL to FNAL}

We repeat our experiments over the wide area networks from BNL to FNAL. Experiment results also verify our claims in previous sections. Correspondingly, Table 8 shows the iperf experiment results in the receiver. Figure 11 gives the comparison of CPU shares. It shows that the networked Linux systems have the serious fairness issues.

\begin{tabular}{|c|c|c|c|c|}
\hline Load & Scheduler & Throughput & $\begin{array}{c}\text { CPU } \\
\text { Share }\end{array}$ & $\begin{array}{c}\text { Reinsertion } \\
\text { Count }\end{array}$ \\
\hline \multirow{2}{*}{ BL0 } & WI & $325 \mathrm{Mbps}$ & $75.877 \%$ & 713 \\
\cline { 2 - 5 } & NI & $304 \mathrm{Mbps}$ & $65.68 \%$ & 0 \\
\hline \multirow{2}{*}{ BL1 } & WI & $277 \mathrm{Mbps}$ & $59.472 \%$ & 593 \\
\cline { 2 - 5 } & NI & $248 \mathrm{Mbps}$ & $47.063 \%$ & 0 \\
\hline \multirow{2}{*}{ BL2 } & WI & $274 \mathrm{Mbps}$ & $58.996 \%$ & 588 \\
\cline { 2 - 5 } & NI & $195 \mathrm{Mbps}$ & $31.922 \%$ & 0 \\
\hline \multirow{2}{*}{ BL4 } & WI & $278 \mathrm{Mbps}$ & $64.144 \%$ & 620 \\
\cline { 2 - 5 } & NI & $116 \mathrm{Mbps}$ & $19.645 \%$ & 0 \\
\hline \multirow{2}{*}{ BL8 } & WI & $273 \mathrm{Mbps}$ & $58.788 \%$ & 586 \\
\cline { 2 - 5 } & NI & $79.8 \mathrm{Mbps}$ & $9.717 \%$ & 0 \\
\hline
\end{tabular}

Table 8 Iperf Experiment Results in the Receiver

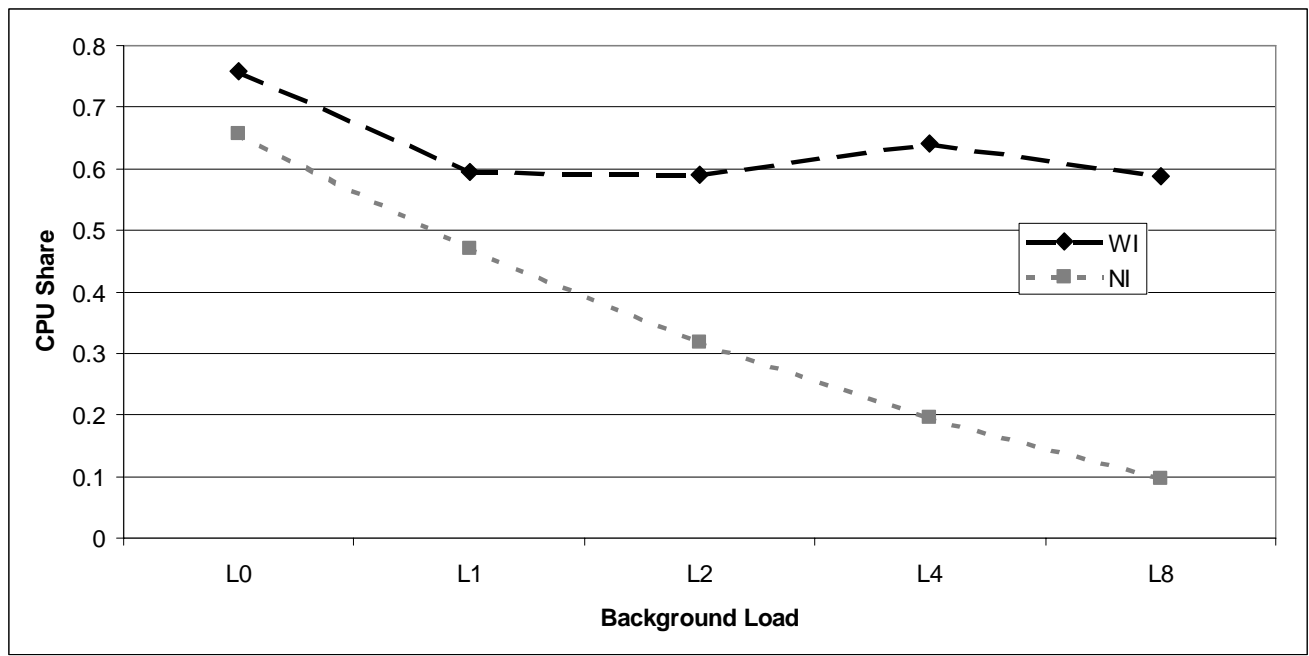

Figure 11 Comparisons of CPU Shares 


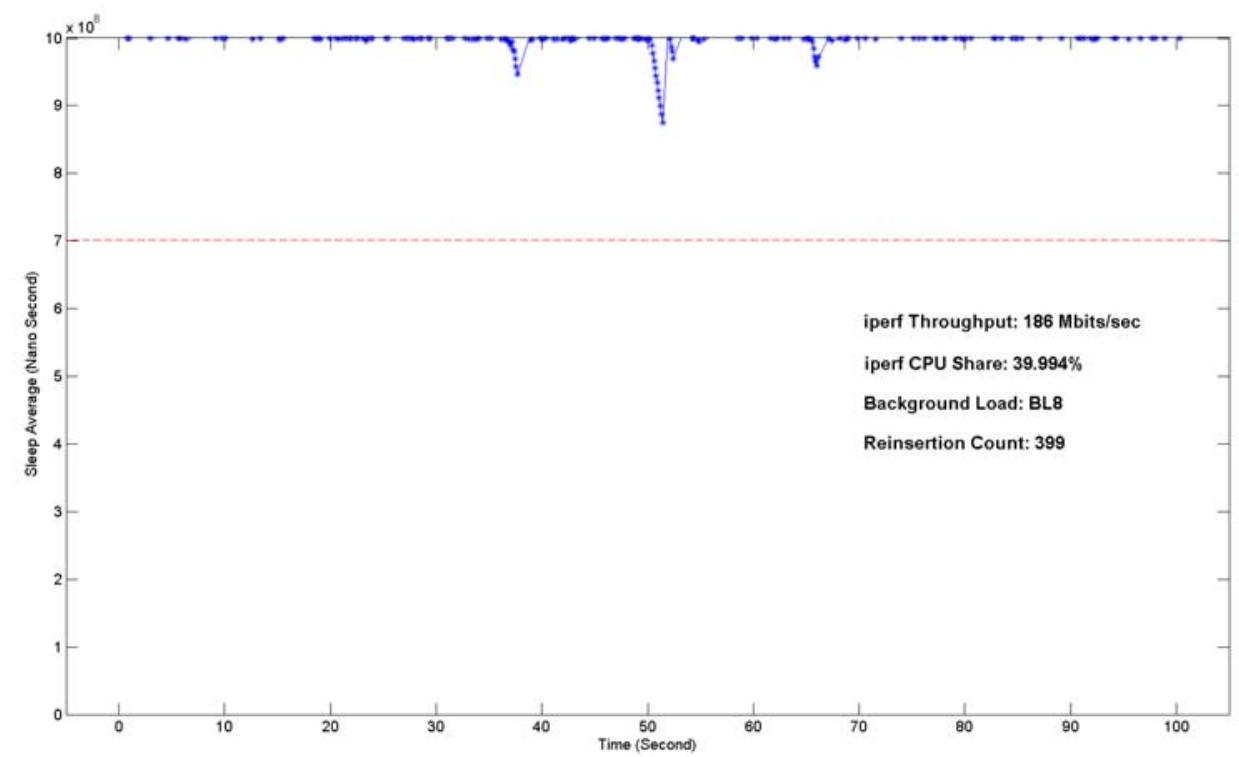

Figure 12 Iperf's sleep_avg in the Receiver

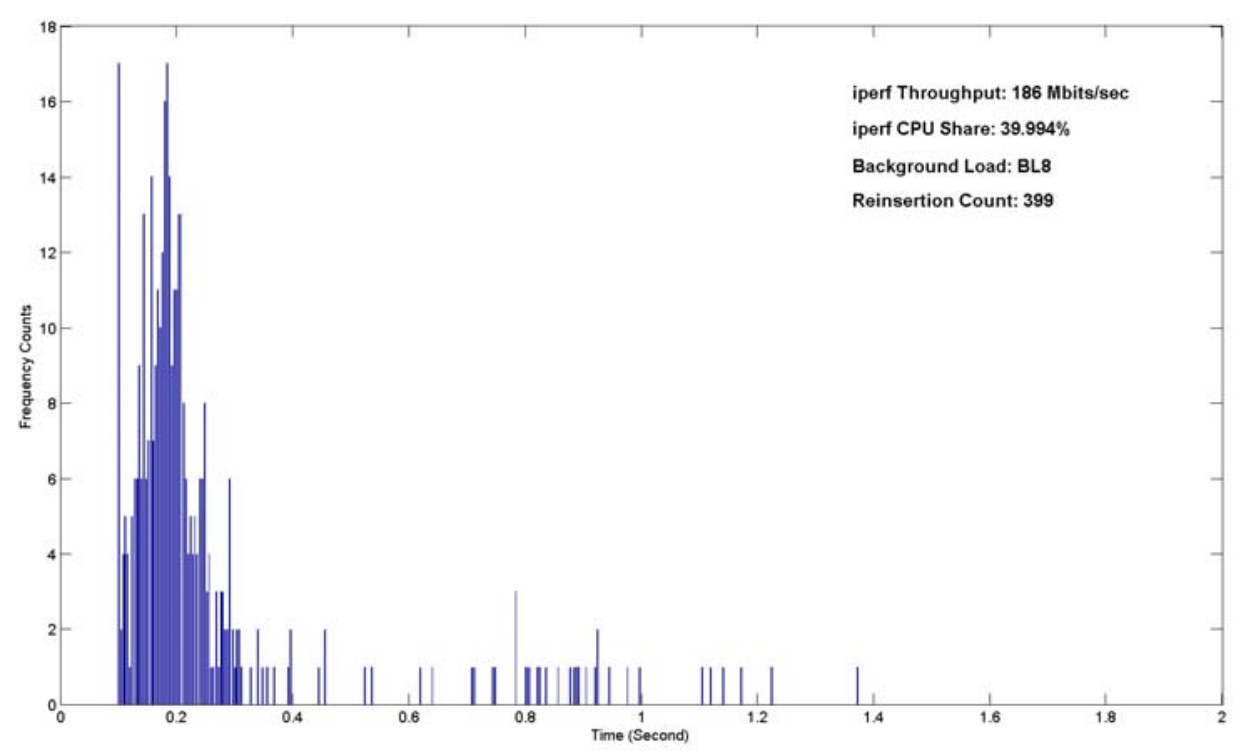

Figure 13 Histogram of Time Intervals between Consecutive Timeslice Expiration Instants for Iperf in the Receiver (WAN)

Figure 12 and 13 give the results of one random wide area network experiment from BNL to FNAL. The background load of the experiment is BL8. Figure 12 gives the iperf's sleep_avg in the receiver. It can be shown that iperf is also categorized as interactive all the time due to network conditions. Correspondingly, Figure 13 shows the histogram of time intervals between consecutive timeslice expiration instants for iperf in the receiver. It gives similar results as Figure 9.

\section{A Possible Solution}

Our experiments and analysis described above have shown that the current interactivity classification mechanism is not effective in distinguishing non-interactive network proc- 
esses from interactive processes, resulting in serious fairness/starvation problems. To summarize, the reasons that cause such consequences are: (1) network packets arrive at the receiver independently and discretely; the "relatively fast" non-interactive network process might frequently sleep to wait for network packets. Though each sleep lasts for a short period of time, the wait-for-packet sleeps occur frequently, more than enough to lead to the interactivity status. (2) The current Linux interactivity mechanism provides the possibilities that a non-interactive network process could consume a high CPU share, and at the same time be incorrectly categorized as "interactive." To resolve the interactivity vs. fairness issue there might be two basic approaches. One approach is to completely overhaul the current Linux interactivity mechanism to eliminate this possibility. However, the current mechanism has been proven effective for traditional non-networked applications. Major modifications would be likely to affect those applications. Clearly, this approach might be complex and time-consuming. The second approach is to reduce or eliminate those sleep_avg updates triggered by short inter-packet sleeps under noninteractive conditions. We pursue the latter course.

\begin{tabular}{|c|c|c|c|c|c|c|c|}
\hline Experiment & $<2 \mathrm{~ms}$ & $<5 \mathrm{~ms}$ & $<10 \mathrm{~ms}$ & $<15 \mathrm{~ms}$ & $<20 \mathrm{~ms}$ & $\begin{array}{c}\text { Through- } \\
\text { put }\end{array}$ & $\begin{array}{c}\text { Mean } \\
(\mathrm{ms})\end{array}$ \\
\hline BNL->FNAL (1) & $68.32 \%$ & $83.82 \%$ & $97.79 \%$ & $99.84 \%$ & $99.88 \%$ & $263 \mathrm{Mbps}$ & 2.2214 \\
\hline BNL->FNAL (2) & $68.72 \%$ & $85.08 \%$ & $98.85 \%$ & $99.92 \%$ & $99.95 \%$ & $221 \mathrm{Mbps}$ & 2.0071 \\
\hline FNAL - > FNAL (1) & $99.78 \%$ & $99.85 \%$ & $99.93 \%$ & $99.93 \%$ & $99.93 \%$ & $383 \mathrm{Mbps}$ & 0.2285 \\
\hline FNAL -> FNAL (2) & $99.70 \%$ & $99.79 \%$ & $99.88 \%$ & $99.88 \%$ & $99.89 \%$ & $438 \mathrm{Mbps}$ & 0.2259 \\
\hline
\end{tabular}

Table 9 wait-for-packet sleep statistics for iperf data transmission experiment

Usually, network applications can be classified into the following categories:

a) Interactive network applications like ssh, telnet, and web browsing. Since those applications involve human interactions, the wait-for-packet sleeps in the receiver usually last for hundreds of milliseconds or even seconds to wait for user's inputs. For example, In [16] Y. Etsion et al. have reported that standard typing at a rate of about 8 characters per second. In the extreme case, if a packet were sent out for each character typed, the inter-packet space would be average around $125 \mathrm{~ms}$.

b) Non-interactive network applications. Some non-interactive network applications, like ftp*, gridftp*, and scp, involve bulk data transmission. As explained above, due to packet-switched network's packet delivery nature: network packets arrive in the receiver independently and discretely. The "relatively fast" network process in the receiver might frequently sleep to wait for network packets. Though each sleep lasts for a relative short period of time, the wait-for-packet sleeps occur so frequently, more than enough to lead to the interactivity status. Iperf also belong to this category. Table 9 gives the wait-for-packet sleep statistics for a group of data transmission experiments in section 5 . It shows that most wait-for-packet sleeps last for a few milliseconds or less.

* FTP implementations usually are multi-processed or multi-threaded: one process/thread is in charge of FTP control channel, which may be interactive; other processes/threads are in charge of data transmissions. Here, we mean FTP's data transmission processes/threads, and similarly for Gridftp. 
c) Multimedia network applications. For these applications, network packets are transmitted and received periodically. For example, VOIP packets are transmitted and received every 20ms. These applications are categorized as "soft real-time" so other measures should be taken, regardless of the issues investigated here, to guarantee their CPU shares and responsiveness. Possibilities include (1) In Linux 2.6, making use of chrt [27] to classify these applications as real-time. Linux 2.6 provides two real-time scheduling policies, SCHED_FIFO and SCHED_RR, which support soft real-time behaviors [1][2]. (2) When developing these applications, specifically requesting real-time support. Linux 2.6 provides a family of systems calls to support such capabilities [2]. However, the main drawback of such approach is that it might reduce application portability [28]. (3) Making use of a proportional-share scheduler [18] [20] to provide protection between various classes of applications. This paper mainly address the interactivity vs. fairness issues for network applications of category (a) and (b).

Table 9 gives us an insight on how to distinguish interactive network applications from non-interactive ones: for a truly interactive application, the wait-for-packet sleeps usually last for tens or hundreds of milliseconds or more; however, the inter-packet sleeps for bulk data transmission applications usually last for a few milliseconds or less. Accordingly, to resolve the interactivity vs. fairness issue in networked Linux systems, our strategy is as follows: when the sleep duration does not exceed some minimal value, sleep_avg for the network process will not be updated; sleep_avg is only updated when the sleep exceeds the threshold. We have modified the Linux kernel, and call this floor value the "Interactive Network Threshold". The value is configurable through a new item in the /proc filesystem, /proc/sys/kernel/interactive_network_threshold, and its unit is milliseconds. It can be set according to the network conditions and the system's purpose. If the system is mainly used for local area networks, a relatively small value such as $5 \mathrm{~ms}$ is quite enough. If the system is used for wide area networks, and the packet jitter is high, interactive_network_threshold could be configured even higher. Usually high packet jitter implies low throughput; it won't cause serious fairness issues in the receiver. Therefore, interactive_network_threshold need not be too high. In our implementation, the default interactive_network_threshold is set at $30 \mathrm{~ms}$. If system owner does not care about the interactivity vs. fairness issues at all, it can be set as 0 . If processing of streaming media such as VOIP is competing with other system loads and has not been protected as suggested above, an interactive_network_threshold of $15 \mathrm{~ms}$ may be better.

We repeat the data transmission experiments as described in section 5 on the Linux updated with the new interactivity parameter described as above. We compare the new experiment data with those obtained in section 5 . The old experiment will be prefixed with "O-“, the new data with "N-“.

Table 10 show the iperf experiment results in the receiver for experiments over Fermilab's sub-networks. Since the fairness issue is not serious with the fast sender, the experiments are run only with the slow sender. The interactive_network_threshold is set as $5 \mathrm{~ms}$. For better comparison and presentation, we show the comparisons of CPU shares in Figure 14. It can be seen that: with the updated interactivity algorithm, iperf's CPU share is effectively decreased when the background load is correspondingly increased; 
the reinsertion count of N-WI is much reduced than that of O-WI. Since interactive_network_threshold is set so low, it won't affect the scheduling of true interactive network applications. The experiment results imply that our proposed solution is effective in resolving the fairness issue with the networked Linux systems while maintaining the interactivity performance for true interactive network applications.

\begin{tabular}{|c|c|c|c|c|}
\hline Load & Scheduler & Throughput & $\begin{array}{c}\text { CPU } \\
\text { Share }\end{array}$ & $\begin{array}{c}\text { Reinsertion } \\
\text { Count }\end{array}$ \\
\hline \multirow{2}{*}{ BL0 } & O-WI & $436 \mathrm{Mbps}$ & $78.489 \%$ & 780 \\
\cline { 2 - 5 } & N-WI & $455 \mathrm{Mbps}$ & $87.467 \%$ & 8 \\
\hline \multirow{2}{*}{ BL1 } & O-WI & $443 \mathrm{Mbps}$ & $81.573 \%$ & 815 \\
\cline { 2 - 5 } & N-WI & $304 \mathrm{Mbps}$ & $56.38 \%$ & 221 \\
\hline \multirow{2}{*}{ BL2 } & O-WI & $438 \mathrm{Mbps}$ & $80.613 \%$ & 801 \\
\cline { 2 - 5 } & $\mathbf{N}-W I$ & $260 \mathrm{Mbps}$ & $47.493 \%$ & 254 \\
\hline \multirow{2}{*}{ BL4 } & O-WI & $430 \mathrm{Mbps}$ & $79.217 \%$ & 785 \\
\cline { 2 - 5 } & $\mathbf{N}-W I$ & $181 \mathrm{Mbps}$ & $32.682 \%$ & 177 \\
\hline \multirow{2}{*}{ BL8 } & $\mathbf{O}-W I$ & $440 \mathrm{Mbps}$ & $81.093 \%$ & 811 \\
\cline { 2 - 5 } & $\mathbf{N}-W I$ & $109 \mathrm{Mbps}$ & $19.748 \%$ & 104 \\
\hline
\end{tabular}

Table 10 Iperf Experiment Results in the Receiver (Slow Sender)

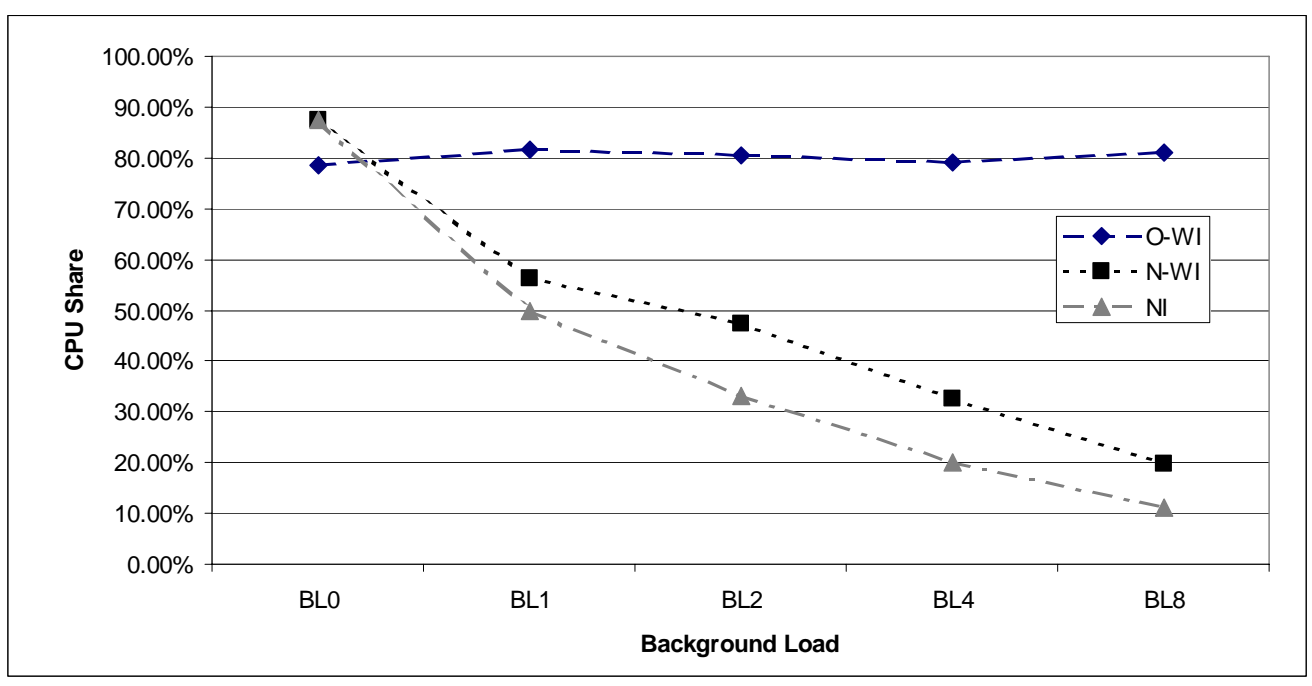

Figure 14 Comparisons of CPU Shares

Figure 15 shows the iperf's sleep_avg in the receiver with the updated interactivity algorithm for a randomly chosen experiment (interactive_network_threshold=5ms, BL8). Compared with Figure 7, it can be seen that most of the time iperf is not categorized as "interactive”. When iperf is not categorized as “interactive”, it won't gain extra runs at the cost of other non-interactive processes. This explains why iperf's CPU share is effectively decreased when the background load is correspondingly increased. It further verifies the effectiveness of our proposed solution. However, it still can be seen from Figure 15 that iperf's sleep_avg might jump from a low value to a relative high value, leading to the interactive status (also in Figure 8). This is caused by the scheduling delay: when a low-dynamic-priority iperf wakes up upon packet arrival, it might wait on the runqueue for a relatively long time before it is scheduled to run, which is fully credited to the sleep_avg. Since the scheduling delays of interactive network processes cannot be 
differentiated from those of non-interactive processes, the influence of this type of scheduling delays is hard to eliminate. This is also the reason that the CPU shares in the N-WI runs are higher than in NI.

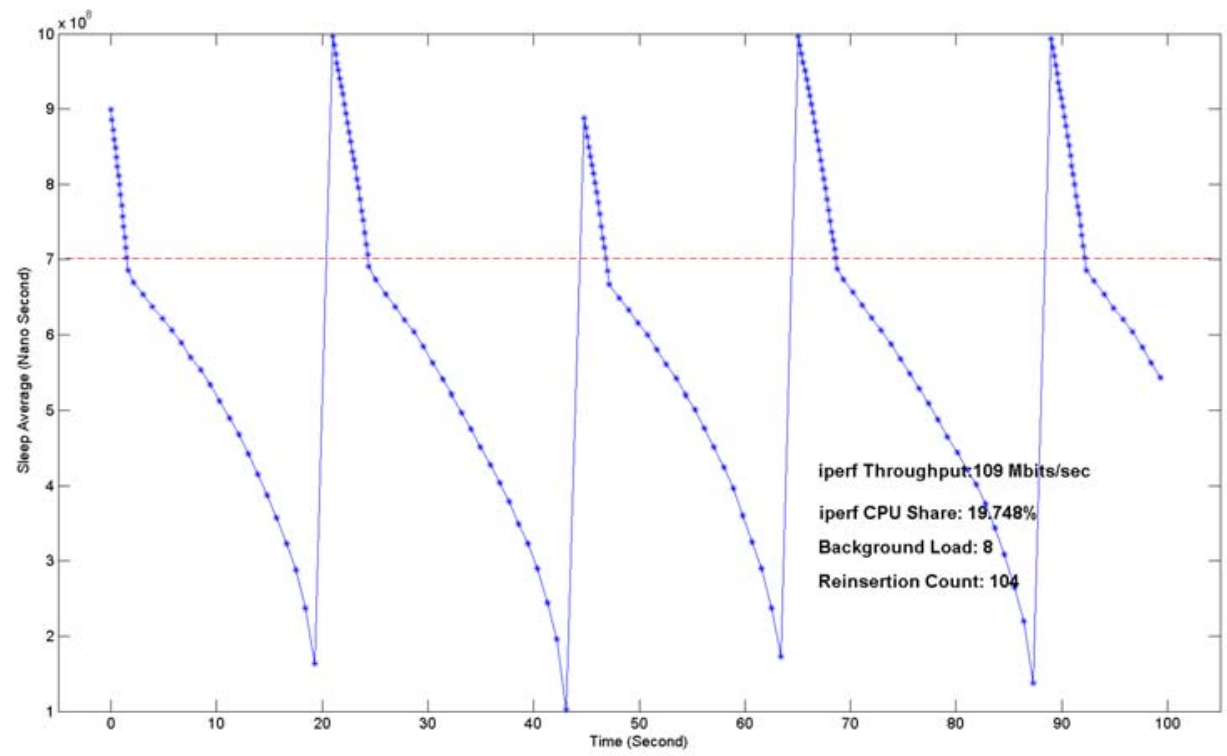

Figure 15 Iperf's sleep_avg in the Receiver (Slow Sender)

Similar results are obtained in experiments over the wide area networks from BNL to FNAL. Figure 16 shows iperf's sleep_avg in the receiver for two random experiments from BNL to FNAL with the new interactivity algorithm. In Figure 16(a), interactive_network_threshold is set as $10 \mathrm{~ms}$, while it is set as $30 \mathrm{~ms}$ in Figure 16(b). It can be seen that for wide area networks, since the packet jitter is higher, the interactive_network_threshold needs to be correspondingly configured higher. Setting interactive_network_threshold to 30ms effectively improves the system's fairness, while not affecting true interactive network applications' performance. In Figure 16, we also see scheduling delays causing jumps in sleep_avg. 


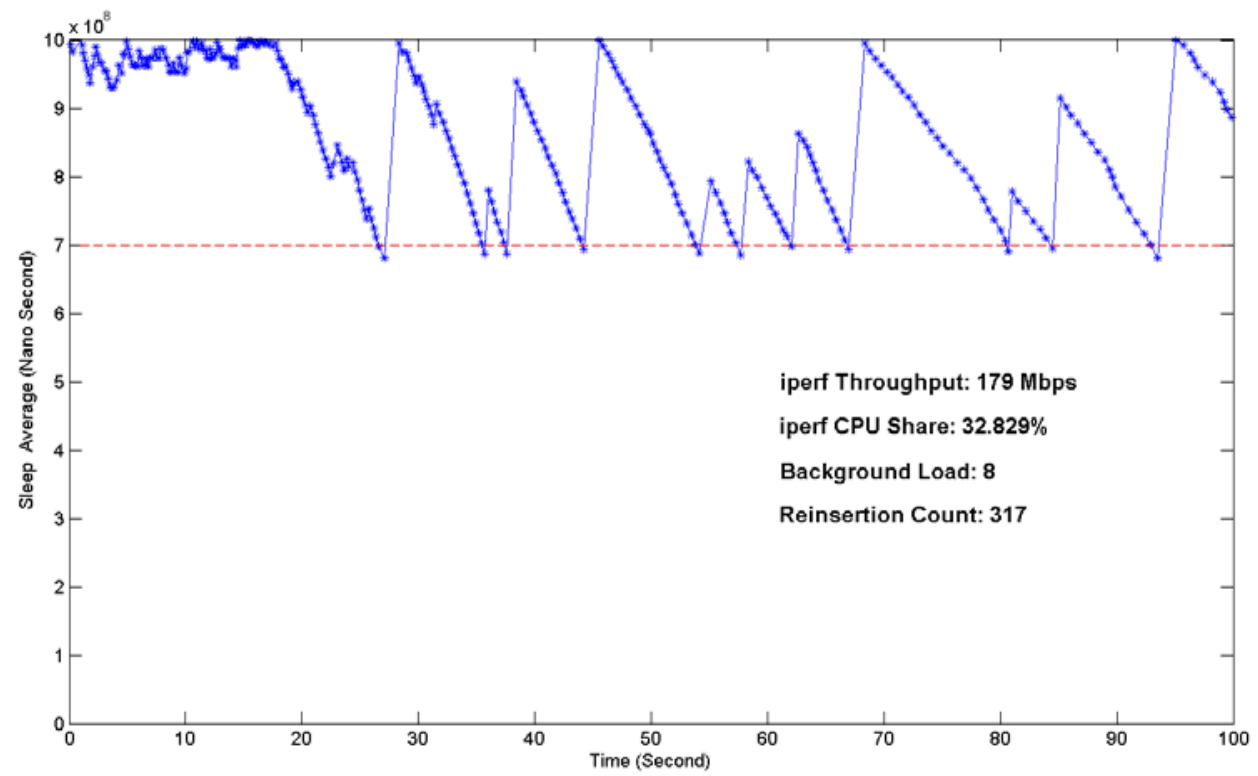

(a) interactive_network_threshold $=10 \mathrm{~ms}$

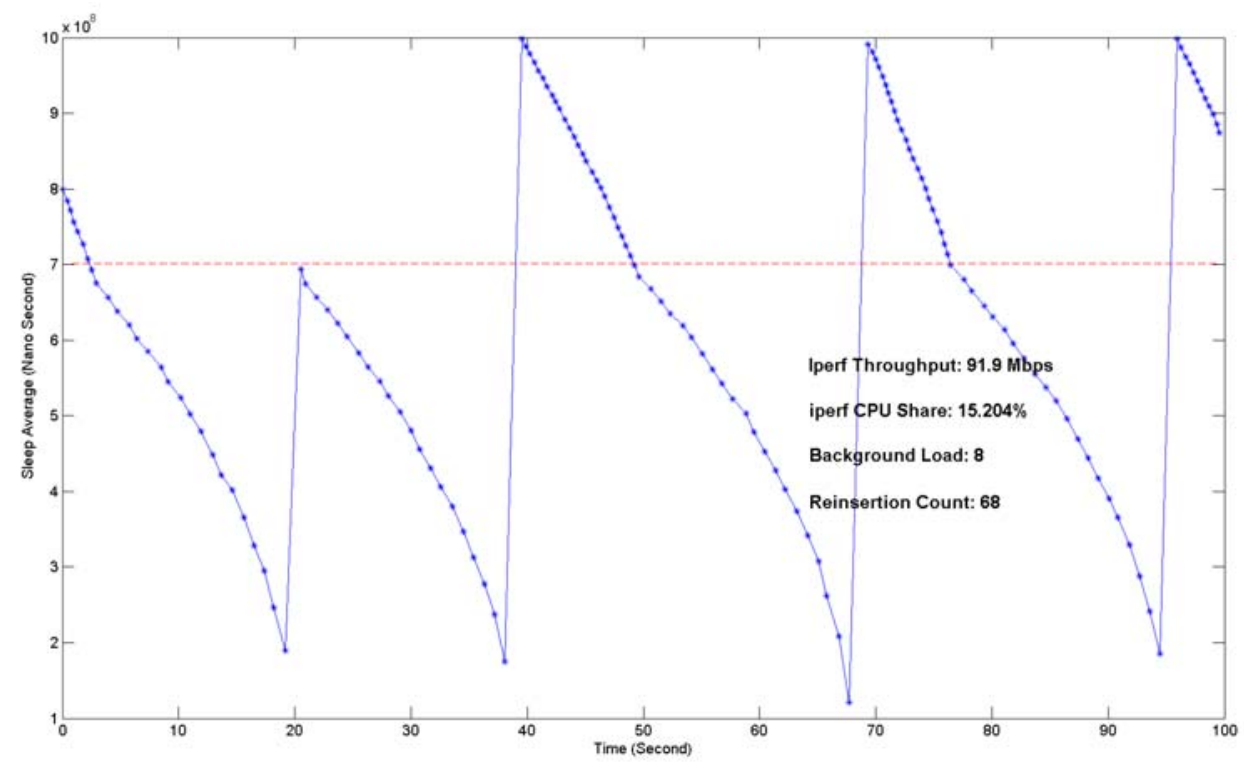

(b) interactive_nework_threshold $=30 \mathrm{~ms}$

Figure 16 Iperf's sleep_avg in the Receiver for Experiments from BNL to FNAL

\section{Conclusions}

Our researches have pointed out that the current Linux interactivity mechanism is not effective in distinguishing non-interactive network processes from interactive network processes, and results in serious fairness/starvation problems. Mathematical analysis and experiments results have verified our conclusions. Further, we propose and test a simple scheduler modification to address the interactivity vs. fairness problems in networked Linux systems. Experiment results have proved the effectiveness of our proposed solu- 
tion. The improvements in fairness come at a cost: the network throughput for a given process may be reduced, while the CPU share, response time, or network throughputs of other processes are improved. This will be a desirable trade-off in some environments, but perhaps not in all.

\section{References}

[1] D. P. Bovet et al., Understanding the Linux Kernel, 3rd Edition, O’Reilly Press, ISBN: 0-59600565-2, 2005.

[2] R. Love, Linux Kernel Development, Second Edition, Novell Press, ISBN: 0672327201, 2005.

[3] C. S. Rodriguez et al., The Linux(R) Kernel Primer: A Top-Down Approach for x86 and PowerPC Architectures, Prentice Hall PTR, ISBN: 0131181637, 2005.

[4] www.kernel.org.

[5] "Goals, Design and Implementation of the new ultra-scalable O(1) scheduler", Linux Documentation, sched-design.txt.

[6] A. Silberschatz et al., Operating System Concepts, 7th Edition, John Wiley \& Sons, ISBN: 0471694665, 2004.

[7] R. Love, "Interactive Kernel Performance: Kernel Performance in Desktop and Real-time Applications," In Proceedings of the Linux Symposium, July 23 - 26, 2003, Ottawa, Canada.

[8] M. Mathis et al., "Web100: Extended TCP Instrumentation for Research, Education and Diagnosis," ACM Computer Communications Review, vol. 33, no. 3, July 2003.

[9] T. Dunigan et al., “A TCP Tuning Daemon”, SuperComputing 2002.

[10] M. Rio et al., "A Map of the Networking Code in Linux Kernel 2.4.20," March 2004.

[11] J. C. Mogul et al., "Eliminating Receive Livelock in an Interrupt-driven Kernel," ACM Transactions on Computer Systems, vol. 15, no. 3, pp. 217--252, 1997.

[12] M. J. Bach, The Design of the UNIX Operating System. Prentice-Hall, ISBN: 0132017997, 1986.

[13] U. Vahalia, UNIX Internals: The New Frontiers, Prentice Hall, ISBN: 0131019082, 1995.

[14] J. Mauro et al., Solaris Internals Core Kernel Architecture, ISBN: 0130224960, 1st Edition, Prentice Hall PTR, 2000.

[15] M. K. McKusick et al., The Design and Implementation of the FreeBSD Operating System, Addison-Wesley Professional, ISBN: 0201702452, 2004.

[16] Y. Etsion et al., "Human-centered Scheduling of Interactive and Multimedia Applications on a Loaded Desktop,” Tech. Rep., Hebrew University, Mar. 2003.

[17] C. A. Waldspurger et al., "Lottery Scheduling: Flexible Proportional-Share Resource Management," In Proceedings of the 1st USENIX Symposium on Operating Systems Design and Implementation, Monterey, CA, November 1994.

[18] P. Goyal et al., "A Hierarchical CPU Scheduler for Multimedia Operating Systems,” In Proceedings of the 2nd OSDI Symposium, October 1996.

[19] J. Nieh et al., "Virtual-time Round-robin: An O(1) Proportional Share Scheduler," In Proceedings of the 2001 USENIX Annual Technical Conference, USENIX, Berkeley, CA, 2001, pp. 245-259.

[20] K. Jeffay et al., "Proportional Share Scheduling of Operating System Services for Real-time Applications,” In IEEE Real Time System Symposium, Madrid, Spain, December 1998.

[21] D. Petrou et al., "Implementing Lottery Scheduling: Matching the Specialisations in Traditional Schedulers,” In Proceedings of the 1999 USENIX Technical Conference, pages 1-14, Monterey, CA, USA, June 1999.

[22] http://kerneltrap.org/node/780

[23] Y. Etsion et al., Effects of clock resolution on the scheduling of real-time and interactive processes, Technical Report 2001-14, Hebrew University, Nov 2001.

[24] J. Davidson, et al., Voice over IP Fundamentals, 1st Edition, Cisco Press, ISBN: 1578701686, 2000.

[25] V. Jacobson, "Congestion Avoidance and Control," In Proceedings of ACM SIGCOMM, Stanford, CA, August 1988, pp. $314-329$.

[26] http://dast.nlanr.net/Projects/Iperf/.

[27] E. Siever et al., Linux in a Nutshell, 5th Edition, O’Reilly Media, Sebastopol, CA, ISBN: 0-59600930-5, 2005. 
[28] Y. Etsion et al., “Desktop Scheduling: How can We Know What the User Wants?” In Proceedings of the 14th international workshop on Network and Operating systems support for Digital Audio and Video, Cork, Ireland, 2004. pp. 110-115. 